QUARTERLY OF APPLIED MATHEMATICS

VOLUME LXXI, NUMBER 4

DECEMBER 2013, PAGES 601-628

S 0033-569X(2013)01277-X

Article electronically published on August 27, 2013

\title{
ASYMPTOTIC EXPANSIONS OF SOLUTIONS OF SYSTEMS OF KOLMOGOROV BACKWARD EQUATIONS FOR TWO-TIME-SCALE SWITCHING DIFFUSIONS
}

\author{
BY \\ DUNG TIEN NGUYEN (Department of Applied Mathematics, Faculty of Applied Science, \\ University of Technology, Ho Chi Minh city, Vietnam) \\ AND \\ G. YIN (Department of Mathematics, Wayne State University, Detroit, Michigan 48202)
}

\begin{abstract}
This work is concerned with systems of coupled partial differential equations (known as Kolmogorov backward equations) for continuous-time Markov processes featuring in the coexistence of continuous dynamics and discrete events. Arising from state-dependent switching diffusions, distinct from the usual Markovian regime-switching systems, the generator of the switching component depends on the continuous state. One of the main ingredients of our models is the two-time-scale formulation. In contrast to the work on Kolmogorov forward equations in the existing literature, new techniques are developed in this paper. Although they originate from probabilistic models, the methods are analytic. Two classes of models, namely, fast-switching systems and fast-diffusion systems, are treated. Under broad conditions, asymptotic expansions are developed for the solutions of the systems of backward equations. These asymptotic series are rigorously justified and error bounds are obtained.
\end{abstract}

1. Introduction. This work is concerned with systems of coupled partial differential equations with two-time scales. They are known as systems of Kolmogorov backward equations, which arise in switching diffusions. The motivation stems from recent advances in the study of regime-switching diffusions and from the needs of emerging applications in wireless communications and financial engineering, where continuous dynamics and discrete events coexist and are intertwined. One of the distinct features is: The jump

Received May 15, 2011.

2010 Mathematics Subject Classification. Primary 34E05, 60J27, 93E20.

Key words and phrases. System of backward equation, two-time scale, asymptotic expansion.

The research of the first author was supported in part by Wayne State University under a Graduate Research Assistantship.

The research of the second author was supported in part by the National Science Foundation under DMS-0603287 and in part by the Air Force Office of Scientific Research under FA9550-10-1-0210.

E-mail address: dungnt@hcmut.edu.vn

E-mail address: gyin@math.wayne.edu

(C) 2013 Brown University

Reverts to public domain 28 years from publication 
process is not Markov, but rather, the switching component has a generator $Q(x, t)$ depending on both the continuous state $x$ and the time $t$. That is, the switching process is not homogeneous in time and is coupled with the continuous dynamics. For some recent results on switching diffusions, we refer the reader to [16.

In many applications, to reduce computational complexity is a major concern. Here, in light of the different rates of changes, we treat two distinct cases, namely, the fastvarying switching and the rapidly-changing diffusion. In the first case, although the discrete component lives in a finite set, the set is rather large owing to various modeling considerations for complex systems and random environments. As a result, one often has to treat a large-dimensional system of partial differential equations. Aiming at reducing the computational complexity, by introducing a two-time-scale formulation, we may divide the large state space of the discrete component to subspaces such that the interactions within each subspace are frequent, but the jumps from one subspace to another are relatively rare. Lumping the states in each subspace into a single super-state leads to a reduced system. Corresponding to the reduced system, the total number of Kolmogorov PDEs is substantially less than that of the original one. Thus, we achieve the goal of reduction of complexity by aggregating states and by taking appropriate averaging. In the second case, the diffusion part has two diffusion processes. One of them is fast varying, whereas the other is slowly changing. Suppose that we are interested in finding the optimal controls of a suitable cost function for this switching diffusion. It is difficult to solve the problem directly due to the different time scales and the interactions of the continuous dynamics with that of the discrete events. Nevertheless, under suitable conditions, the fast-varying diffusion does not blow up, but it has an invariant measure. As a result, it may be viewed as a noise and can be averaged out with respect to the invariant measure leading to a limit system. We can proceed to use the optimal control of the limit system (assuming that it has an optimal control) to construct controls of the original system. This leads to near-optimal controls of the original systems with reduced computational effort.

For both cases, to circumvent the difficulty, we realize that not all "states" change at the same speed. Some of them evolve in a fast pace and the others change slowly. An effective way of handling the underlying systems is to successfully bring out the intrinsic time-scale structure. In both cases, a central issue is that the limit turns out to be an average with respect to invariant measures. For some of the recent work on two-time-scale modeling using diffusions without switching, we refer the reader to 4, where stochastic volatility was modeled by use of the fast-slow diffusions. Most recent results dealing with backward equations of diffusions without switching can be found in [7. The works on systems of forward equations for switching diffusions can be found in [5, 6].

In this paper, our approach is constructive. Not only do asymptotic series provide the convergence to the solution but also rates of convergence together with uniform error bounds. The novelty of the current paper compared with [5, 6] includes the following aspects. (1) In both [5, 6], the switching takes place in an irreducible finite set, whereas the switching is allowed to evolve in several irreducible classes in this paper. (2) The solutions of the forward equations are probability measures, whereas those of the backward equations are functionals. To facilitate the analysis, new techniques are developed 
in this paper. (3) For the forward equations, the probabilistic nature enables us to use the orthogonality (with respect to the invariant measure) directly, whereas in this paper, we need to bring out certain orthogonality from tangled information. We note that the asymptotic expansions constructed will be of utility for many control and optimization problems of large-scale and complex systems.

To proceed, the rest of the paper is arranged as follows. Section 2 starts with the formulation of the problem. Section 3 is concerned with systems of backward equations involving fast-varying switching processes. That is, compared with the continuous dynamics, the discrete component evolves an order of magnitude faster than that of the continuous counterpart. In Section 4, we consider the case that the diffusion component varies much faster than the discrete events and becomes the dominating force. For both Sections 3 and 4, we construct asymptotic expansions of the solutions of systems of backward equations. Our constructive methods provide us with a step-by-step procedure. After obtaining the formal asymptotic expansions, we derive the error bounds. This enables us to show that the asymptotic series so constructed are uniformly valid with the desired uniform error bounds. Section 5 provides illustrations of our results and makes additional remarks to conclude the paper.

2. Problem formulation. Consider a switching diffusion that is a Markov process $Y(t)$ having two components, a continuous component $X(t)$ and a switching component $\alpha(t)$. We assume $X(t)$ and $\alpha(t)$ take values in a compact set and a finite set, respectively. For simplicity, we take $X(t)$ to be one dimensional and the compact set to be a unit circle. The state space of the process $Y(t)=(X(t), \alpha(t))$ is $\mathcal{X}=\mathcal{S} \times \mathcal{M}$, where $\mathcal{S}$ is the unit circle and $\mathcal{M}=\{1, \ldots, m\}$. By identifying the endpoints 0 and 1 , let $x \in[0,1]$ be the coordinates in $\mathcal{S}$. Suppose $b(\cdot, \cdot, \cdot):[0,1] \times \mathcal{M} \times[0, T] \mapsto \mathbb{R}$ and $\sigma(\cdot, \cdot, \cdot):[0,1] \times \mathcal{M} \times$ $[0, T] \mapsto \mathbb{R}$. The dynamics of the process can be represented by the following stochastic differential equation:

$$
d X(t)=b(X(t), \alpha(t), t) d t+\sigma(X(t), \alpha(t), t) d B(t),
$$

together with a transition law for the second component $\alpha(t)$,

$$
P(\alpha(t+\Delta)=\ell \mid \alpha(t)=k, X(t)=x)=q_{k \ell}(x, t) \Delta+o(\Delta), k \in \mathcal{M},
$$

where $o(\Delta) / \Delta \rightarrow 0$ as $\Delta \rightarrow 0$. In the above, $B(\cdot)$ is a standard real-valued Brownian motion, and $Q(x, t)=\left(q_{k \ell}(x, t)\right)$ is an $x$ and $t$ dependent generator for the switching process satisfying for each $k, \ell \in \mathcal{M}$ and $k \neq \ell, q_{k \ell}(x, t) \geq 0$, and for each $k \in \mathcal{M}$, $\sum_{\ell \in \mathcal{M}} q_{k \ell}(x, t)=0$. In this paper, the symbol " " is reserved for the transpose of a vector or a matrix throughout the paper. Associated with (2.1) and (2.2), there is an operator $\mathcal{L}$ defined by

$$
\begin{aligned}
& \mathcal{L}(x, t) u(x, t)=\left(\mathcal{L}_{1}(x, t) u(x, 1, t), \ldots, \mathcal{L}_{l}(x, t) u(x, m, t)\right)^{\prime}, \text { where } \\
& \mathcal{L}_{k}(x, t) u(x, k, t)=\frac{1}{2} a(x, k, t) \frac{\partial^{2}}{\partial x^{2}} u(x, k, t)+b(x, k, t) \frac{\partial}{\partial x} u(x, k, t), \quad k \in \mathcal{M},
\end{aligned}
$$


$u(x, k, t)$ is a real-valued function for each $k \in \mathcal{M}, u(x, t)=(u(x, 1, t), \ldots, u(x, m, t))^{\prime} \in$ $\mathbb{R}^{m}$, and $a(x, k, t)=\sigma^{2}(x, k, t), k \in \mathcal{M}$. Consider the following system of equations:

$$
\begin{aligned}
& -\frac{\partial}{\partial t} u(x, k, t)=\mathcal{L}_{k}(x, t) u(x, k, t)+Q(x, t) u(x, \cdot, t)(k), k \in \mathcal{M}, \\
& u(x, k, T)=g(x, k), k \in \mathcal{M},
\end{aligned}
$$

where for each $k=1, \ldots, m, u(\cdot, k, \cdot) \in C^{2,1}([0,1] \times[0, T])$ (twice continuously differentiable with respect to $x$ and continuously differentiable with respect to $t$ ), and

$$
Q(x, t) u(x, \cdot, t)(k)=\sum_{\ell \in \mathcal{M}} q_{k \ell}(x, t) u(x, \ell, t) .
$$

System (2.4) is the well-known system of Kolmogorov backward equations.

3. Rapid switching. Let $\varepsilon>0$ be a small parameter, $\alpha^{\varepsilon}(\cdot)$ be a jump process with state space $\mathcal{M}$ and $Q(x, t)$ be of the form $Q^{\varepsilon}(x, t)=\frac{\widetilde{Q}(x, t)}{\varepsilon}+\widehat{Q}(x, t)$. Henceforth, we relabel the states of $\mathcal{M}$ so that $\mathcal{M}=\mathcal{M}_{1} \cup \mathcal{M}_{2} \cup \cdots \cup \mathcal{M}_{l} \cup \mathcal{M}_{*}$, where $\mathcal{M}_{\imath}=$ $\left\{s_{\imath 1}, \ldots, s_{\imath m_{\imath}}\right\}$, for $\iota=1, \ldots, l$ and $\mathcal{M}_{*}=\left\{s_{* 1}, \ldots, s_{* m_{*}}\right\}$. In what follows, we will use $s_{\imath \jmath}$ with $\imath=1, \ldots, l, *$ and $\jmath=1, \ldots, m_{\imath}$ to denote a state in $\mathcal{M}$, and we often use $k \in \mathcal{M}$ to indicate that $k$ is one of the $s_{\imath \jmath}$ 's. This convention will be used throughout. Assume that $\widetilde{Q}(x, t)$ is of the form:

$$
\widetilde{Q}(x, t)=\left(\begin{array}{cccc}
\widetilde{Q}^{1}(x, t) & & & \\
& \ddots & & \\
& & \widetilde{Q}^{l}(x, t) & \\
\widetilde{Q}_{*}^{1}(x, t) & \ldots & \widetilde{Q}_{*}^{l}(x, t) & \widetilde{Q}_{*}(x, t)
\end{array}\right) .
$$

Denote

$$
\begin{aligned}
& u^{\varepsilon}(x, t)=\left(u^{\varepsilon}\left(x, s_{\imath \jmath}, t\right): s_{\imath \jmath} \in \mathcal{M}, \imath=1, \ldots, l, *, \jmath=1, \ldots, m_{\imath}\right)^{\prime}, \\
& g(x)=\left(g\left(x, s_{\imath \jmath}\right): s_{\imath \jmath} \in \mathcal{M}, \imath=1, \ldots, l, *, \jmath=1, \ldots, m_{\imath}\right)^{\prime} .
\end{aligned}
$$

Then system (2.4) has the form

$$
-\frac{\partial}{\partial t} u^{\varepsilon}(x, t)=\mathcal{L}(x, t) u^{\varepsilon}(x, t)+Q^{\varepsilon}(x, t) u^{\varepsilon}(x, t), \quad u^{\varepsilon}(x, T)=g(x) .
$$

We make the following assumptions.

(A1) For each $\imath=1, \ldots, l, t \in[0, T]$, and each $x \in[0,1], \widetilde{Q}^{\imath}(x, t)$ is weakly irreducible in that for any $\imath=1, \ldots, l$ and $x \in[0,1], \nu^{\imath}(x, t) \widetilde{Q}^{\imath}(x, t)=0, \nu^{\imath}(x, t) \mathbb{1}_{m_{\imath}}=$ $\sum_{\jmath=1}^{m_{\imath}} \nu_{\jmath}^{\imath}(x, t)=1$ has a unique solution, which is termed a quasi-stationary distribution. Denote $\nu^{\imath}(x, t)=\left(\nu_{1}^{\imath}(x, t), \nu_{2}^{\imath}(x, t), \ldots, \nu_{m_{\imath}}^{\imath}(x, t)\right) \in \mathbb{R}^{m_{\imath}}$ and $\mathbb{1}_{m_{\imath}}=$ $(1, \ldots, 1)^{\prime} \in \mathbb{R}^{m_{\imath}}$.

(A2) For some positive integer $n, \widetilde{Q}(\cdot, \cdot)$ and $\widehat{Q}(\cdot, \cdot) \in C^{2(n+2), n+2}([0,1] \times[0, T])$. That is, $\widetilde{Q}(\cdot, \cdot)$ and $\widehat{Q}(\cdot, \cdot)$ are $2(n+2)$-times continuously differentiable with respect to $x$ and $(n+2)$-times continuously differentiable with respect to $t$.

(A3) For each $t \in[0, T]$ and $x \in[0,1], \widetilde{Q}_{*}(x, t)$ is Hurwitz (i.e., all of its eigenvalues have negative real parts).

(A4) For each $k \in \mathcal{M}, g(\cdot, k)$ are periodic in $x$ with period 1 and $g(\cdot, k) \in C^{2(n+2)}([0,1])$. 
(A5) For each $k \in \mathcal{M}, a(\cdot, k, t), b(\cdot, k, t)$ are periodic in $x$ with period 1 for all $t \in[0, T]$ and $a(\cdot, k, \cdot), b(\cdot, k, \cdot) \in C^{2(n+2), n+2}([0,1] \times[0, T])$.

3.1. Construction of asymptotic expansions. For convenience, we use a stretched variable

$$
\tau=\frac{T-t}{\varepsilon}
$$

which magnifies the details of the solution near the terminal time $T$. Denote

$$
\begin{aligned}
& \Phi_{n}^{\varepsilon}(x, t)=\left(\Phi_{n}^{\varepsilon}(x, k, t): k=s_{\imath \jmath}, \imath=1, \ldots, l, *, \jmath=1, \ldots, m_{\imath}\right)^{\prime} \\
& \Psi_{n}^{\varepsilon}(x, \tau)=\left(\Psi_{n}^{\varepsilon}(x, k, t): k=s_{\imath \jmath}, \imath=1, \ldots, l, *, \jmath=1, \ldots, m_{\imath}\right)^{\prime} .
\end{aligned}
$$

We aim to approximate the solution $u^{\varepsilon}(x, t)$ of (3.6) by

$$
\begin{aligned}
& \Phi_{n}^{\varepsilon}(x, t)+\Psi_{n}^{\varepsilon}(x, \tau), \quad \text { where } \\
& \Phi_{n}^{\varepsilon}(x, t)=\sum_{j=0}^{n} \varepsilon^{j} \phi_{j}(x, t), \quad \Psi_{n}^{\varepsilon}(x, \tau)=\sum_{j=0}^{n} \varepsilon^{j} \psi_{j}(x, \tau) .
\end{aligned}
$$

In constructing the asymptotic expansions, to obtain the desired estimates, we need to compute a couple of more terms. Substituting $\Phi_{i}^{\varepsilon}(x, t)$ for $i=0, \ldots, n+2$ into (2.4) and equating coefficients of powers of $\varepsilon^{i}$, we obtain:

$$
\begin{aligned}
& \widetilde{Q}(x, t) \phi_{0}(x, t)=0, \\
& \widetilde{Q}(x, t) \phi_{1}(x, t)=-\frac{\partial}{\partial t} \phi_{0}(x, t)-(\mathcal{L}+\widehat{Q})(x, t) \phi_{0}(x, t), \\
& \ldots \ldots \\
& \widetilde{Q}(x, t) \phi_{i+1}(x, t)=-\frac{\partial}{\partial t} \phi_{i}(x, t)-(\mathcal{L}+\widehat{Q})(x, t) \phi_{i}(x, t),
\end{aligned}
$$

for $i=1, \ldots, n+2$, where $\mathcal{L}(x, t) \phi_{i}(x, t)=\left(\mathcal{L}_{k}(x, t) \phi_{i}(x, k, t): k=s_{\imath \jmath}, \imath=1, \ldots, l, *, \jmath=\right.$ $\left.1, \ldots, m_{\imath}\right)^{\prime}$. Likewise, substituting $\Psi_{i}(x, \tau)$ for $i \leq n+2$ into (3.6), we obtain

$$
\frac{\partial}{\partial \tau}\left(\sum_{j=0}^{i} \varepsilon^{j} \psi_{j}(x, \tau)\right)=\sum_{j=0}^{i} \varepsilon^{j}(\widetilde{Q}(x, T-\varepsilon \tau)+\varepsilon(\mathcal{L}+\widehat{Q})(x, T-\varepsilon \tau)) \psi_{j}(x, \tau) .
$$

For simplicity, we denote the $j$ th-order partial derivative w.r.t. $t$ by $f^{(j)}(x, t)=\frac{\partial^{j} f(x, t)}{\partial t^{j}}$ in what follows. By means of the Taylor expansion, we have

$$
\begin{aligned}
\widetilde{Q}(x, T-\varepsilon \tau) & =\sum_{j=0}^{i} \frac{\widetilde{Q}^{(j)}(x, T)}{j !}(-\varepsilon \tau)^{j}+\widetilde{R}_{i}(x, \varepsilon \tau), \\
\varepsilon(\mathcal{L}+\widehat{Q})(x, T-\varepsilon \tau) & =\sum_{j=0}^{i-1} \frac{(\mathcal{L}+\widehat{Q})^{(j)}(x, T)}{j !} \varepsilon(-\varepsilon \tau)^{j}+\widehat{R}_{i-1}(x, \varepsilon \tau),
\end{aligned}
$$

where $\widetilde{R}_{i}(x, \varepsilon \tau)=O\left(\varepsilon^{i+1}\right)$ and $\widehat{R}_{i-1}(x, \varepsilon \tau)=O\left(\varepsilon^{i+1}\right)$ uniformly in $x \in[0,1]$ for any $\tau>0$. Equating coefficients of powers of $\varepsilon^{i}$, for $i=0,1, \ldots, n+2$ and using the Taylor 
expansions above, we obtain

$$
\begin{aligned}
& \frac{\partial \psi_{0}(x, \tau)}{\partial \tau}=\widetilde{Q}(x, T) \psi_{0}(x, \tau), \\
& \frac{\partial \psi_{1}(x, \tau)}{\partial \tau}=\widetilde{Q}(x, T) \psi_{1}(x, \tau)+\left(-\tau \widetilde{Q}^{(1)}(x, T)+(\mathcal{L}+\widehat{Q})(x, T)\right) \psi_{0}(x, \tau), \\
& \cdots \cdots \\
& \frac{\partial \psi_{i}(x, \tau)}{\partial \tau}=\widetilde{Q}(x, T) \psi_{i}(x, \tau)+r_{i}(x, \tau), \\
& r_{i}(x, \tau)=\sum_{j=0}^{i-1}\left((-\tau)^{i-j} \frac{\widetilde{Q}^{(i-j)}(x, T)}{(i-j) !}+(-\tau)^{i-j-1} \frac{(\mathcal{L}+\widehat{Q})^{(i-j-1)}(x, T)}{(i-j-1) !}\right) \psi_{j}(x, \tau) .
\end{aligned}
$$

From the terminal condition, we derive $\phi_{0}(x, T)+\psi_{0}(x, 0)=g(x)$ and $\phi_{i}(x, T)+\psi_{i}(x, 0)$ $=0$, for $i>0$. Therefore, we obtain

$$
\begin{aligned}
& \psi_{0}(x, \tau)=\exp (\widetilde{Q}(x, T) \tau)\left(g(x)-\phi_{0}(x, T)\right), \\
& \psi_{i}(x, \tau)=-\exp (\widetilde{Q}(x, T) \tau) \phi_{i}(x, T)+\int_{0}^{\tau} \exp (\widetilde{Q}(x, T)(\tau-s)) r_{i}(x, s) d s, \text { for } i>0 .
\end{aligned}
$$

Denote

$$
\widetilde{\mathbb{1}}(x, t)=\left(\begin{array}{ccc}
\mathbb{1}_{m_{1}} & & \\
& \ddots & \\
& & \mathbb{1}_{m_{l}} \\
d^{1}(x, t) & \ldots & d^{l}(x, t)
\end{array}\right),
$$

where $d^{\imath}(x, t)=-\widetilde{Q}_{*}^{-1}(x, t) \widetilde{Q}_{*}^{\imath}(x, t) \mathbb{1}_{m_{\imath}}$, for $\imath=1, \ldots, l$. In what follows, we will prove the smoothness of $\varphi_{i}$ for $0 \leq i \leq n+2$ and the exponential decay of $\psi_{i}$ for $0 \leq i \leq n+1$, which implies the desired error bound by Lemma 3.9.

LEMma 3.1. The solutions of the equation $\widetilde{Q}(x, t) \phi(x, t)=0$ are given by $\phi(x, t)=$ $\widetilde{\mathbb{1}}(x, t) \beta(x, t)$, with $\beta(x, t)=\left(\beta^{1}(x, t), \ldots, \beta^{l}(x, t)\right)^{\prime} \in \mathbb{R}^{l}$. More precisely, $\phi(x, t)$ is of the partitioned form $\phi(x, t)=\left(\left[\phi^{1}(x, t)\right]^{\prime}, \ldots,\left[\phi^{l}(x, t)\right]^{\prime},\left[\phi^{*}(x, t)\right]^{\prime}\right)^{\prime}$ such that $\phi^{\imath}(x, t) \in \mathbb{R}^{m_{\imath} \times 1}$ and $\phi^{*}(x, t) \in \mathbb{R}^{m_{*} \times 1}$ satisfy $\phi^{\imath}(x, t)=\beta^{\imath}(x, t) \mathbb{1}_{m_{\imath}}$ and $\phi^{*}(x, t)=$ $\sum_{\imath=1}^{l} \beta^{\imath}(x, t) d^{\imath}(x, t)$.

Proof. Let $\phi(x, t)=\left(\left[\phi^{1}(x, t)\right]^{\prime}, \ldots,\left[\phi^{l}(x, t)\right]^{\prime},\left[\phi^{*}(x, t)\right]^{\prime}\right)^{\prime}$ be a solution of the above equation. Then for any $\imath=1, \ldots, l, \widetilde{Q}^{\imath}(x, t) \phi^{\imath}(x, t)=0$ and $\sum_{\imath=1}^{l} \widetilde{Q}_{*}^{\imath}(x, t) \phi^{\imath}(x, t)+$ $\widetilde{Q}_{*}(x, t) \phi^{*}(x, t)=0$. Thus $\phi^{*}(x, t)=\sum_{\imath=1}^{l}-\beta^{\imath}(x, t) \widetilde{Q}_{*}^{-1}(x, t) \widetilde{Q}_{*}^{\imath}(x, t) \mathbb{1}_{m_{\imath}}$ and $\phi^{\imath}(x, t)=$ $\beta^{\imath}(x, t) \mathbb{1}_{m_{\imath}}$. The lemma is proved.

Denote

$$
\nu(x, t)=\left(\operatorname{diag}\left(\nu^{1}(x, t), \ldots, \nu^{l}(x, t), 0_{l \times m_{*}}\right)\right)=\left(\begin{array}{cccc}
\nu^{1}(x, t) & & & 0_{1 \times m_{*}} \\
& \ddots & & \vdots \\
& & \nu^{l}(x, t) & 0_{1 \times m_{*}}
\end{array}\right),
$$




$$
\bar{P}(x)=\widetilde{\mathbb{1}}(T) \nu(x, T)=\left(\begin{array}{cccc}
\mathbb{1}_{m_{1}} \nu^{1}(x, T) & & \\
& \ddots & & \\
& & \mathbb{1}_{m_{l}} \nu^{l}(x, T) & \\
d^{1}(x, T) \nu^{1}(x, T) & \ldots & d^{l}(x, T) \nu^{l}(x, T) & 0_{m_{*} \times m_{*}}
\end{array}\right) .
$$

Lemma 3.2. For each $\imath=1, \ldots, l$, suppose that $\widetilde{Q}^{\imath}(x, T)$ is weakly irreducible. Then there exist constants $C$ and $\gamma$ such that $\left|\exp \left(\widetilde{Q}^{\imath}(x, T) \tau\right)-\mathbb{1}_{m_{\imath}} \nu^{\imath}(x, T)\right| \leq C e^{-\gamma \tau}$.

Proof. See [14, Lemma A.2].

Lemma 3.3. There exist positive constants $\gamma$ and $C$ such that

$$
|\exp (\widetilde{Q}(x, T) \tau)-\bar{P}(x)| \leq C e^{-\gamma \tau}, \text { for all } \tau,
$$

where $|A|$ is the matrix norm (e.g., $|A|=\max _{1 \leq \iota \leq m} \sum_{\ell=1}^{m}\left|a_{\iota \ell}\right|$ ).

Proof. It suffices to show for all $z \in \mathbb{R}^{n \times 1},|[\exp (\widetilde{Q}(x, T) \tau)-\bar{P}(x)] z| \leq C e^{-\gamma \tau}|z|$. Given $z=\left(z^{1}, \ldots, z^{l}, z^{*}\right)^{\prime} \in \mathbb{R}^{m \times 1}$, set $y(x, \tau)=\left(y^{1}(x, \tau), \ldots, y^{l}(x, \tau), y^{*}(x, \tau)\right)^{\prime}=$ $\exp (\widetilde{Q}(x, T) \tau) z$. Then

$$
\bar{P}(x) z=\left(\begin{array}{c}
\mathbb{1}_{m_{1}} \nu^{1}(x, T) z^{1} \\
\vdots \\
\mathbb{1}_{m_{l}} \nu^{1}(x, T) z^{l} \\
\sum_{\imath=1}^{l} d^{\imath}(x, T) \nu^{\imath}(x, T) z^{\imath}
\end{array}\right)
$$

and $y(x, \tau)$ is a solution to

$$
\frac{d y(x, \tau)}{d \tau}=\widetilde{Q}(x, T) y(x, \tau), \quad y(x, 0)=z
$$

It follows that

$$
\frac{d y^{*}(x, \tau)}{d \tau}=\widetilde{Q}_{*}(s, T) y^{*}(x, \tau)+\sum_{\imath=1}^{l} \widetilde{Q}_{*}^{\imath}(x, T) y^{\imath}(x, \tau), \quad y^{*}(x, 0)=z^{*},
$$

and for $\imath=1, \ldots, l$,

$$
\frac{d y^{\imath}(x, \tau)}{d \tau}=\widetilde{Q}^{\imath}(x, T) y^{\imath}(x, \tau), \quad y^{\imath}(x, 0)=z^{\imath} .
$$

Then $y^{*}(x, \tau)=\exp \left(\widetilde{Q}_{*}(x, T) \tau\right) z^{*}+\sum_{\imath=1}^{l} \int_{0}^{\tau} \exp \left(\widetilde{Q}_{*}(x, T)(\tau-s)\right) \widetilde{Q}_{*}^{\imath}(x, T) y^{\imath}(x, s) d s$, and for each $\imath=1, \ldots, l, y^{\imath}(x, \tau)=\exp \left(\widetilde{Q}^{\imath}(x, T) \tau\right) z^{\imath}$. By Lemma 3.2,

$$
\left|y^{\imath}(x, \tau)-\mathbb{1}_{m_{\imath}} \nu^{\imath}(x, T) z^{\imath}\right|=\left|\exp \left(\widetilde{Q}^{\imath}(x, T) \tau\right)-\mathbb{1}_{m_{\imath}} \nu^{\imath}(x, T)\right|\left|z^{\imath}\right| \leq C \mathrm{e}^{-\gamma \tau}|z| .
$$

Since $\widetilde{Q}_{*}(x, \tau)$ is a Hurwitz matrix,

$$
\widetilde{Q}_{*}^{-1}(x, T)=-\int_{0}^{\infty} \exp \left(\widetilde{Q}_{*}(x, T) s\right) d s=-\int_{0}^{\tau} \exp \left(\widetilde{Q}_{*}(x, T)(\tau-s)\right) d s-\int_{\tau}^{\infty} \exp \left(\widetilde{Q}_{*}(x, T) s\right) d s .
$$


Therefore,

$$
\begin{aligned}
& y^{*}(x, \tau)-\sum_{\imath=1}^{l} d^{\imath}(x, T) \nu^{\imath}(x, T) z^{\imath}=\exp \left(\widetilde{Q}_{*}(x, T) \tau\right) z^{*} \\
& +\sum_{\imath=1}^{l} \int_{0}^{\tau} \exp \left(\widetilde{Q}_{*}(x, T)(\tau-s)\right) \widetilde{Q}_{*}^{\imath}(x, T) y^{\imath}(x, s) d s+\sum_{\imath=1}^{l} \widetilde{Q}_{*}^{-1}(x, T) \widetilde{Q}_{*}^{\imath}(x, T) \mathbb{1}_{m_{\imath}} \nu^{\imath}(x, T) z^{\imath} .
\end{aligned}
$$

Denote the first two terms by $A_{0}$ and $A_{\infty}$ respectively, and denote each element of the sum in the last term by $A_{\imath}$ for $\iota=1, \ldots, l$ respectively. Then $\left|A_{0}\right| \leq C \mathrm{e}^{-\gamma \tau}|z|$ and $\left|A_{\infty}\right| \leq C \int_{\tau}^{\infty} \mathrm{e}^{-\gamma s}|z| d s=C \mathrm{e}^{-\gamma \tau}|z|$. Moreover, owing to Lemma 3.2, for each $\imath=$ $1, \ldots, l,\left|A_{\imath}\right| \leq C|z| \int_{0}^{\tau} \mathrm{e}^{-\gamma(\tau-s)} \mathrm{e}^{-\gamma s} d s=C \tau \mathrm{e}^{-\gamma \tau}|z| \leq C \mathrm{e}^{-\widetilde{\gamma} \tau}|z|$, for some $0<\widetilde{\gamma}<\gamma$. These inequalities lead to the desired result.

3.2. Leading term $\phi_{0}(x, t)$ and zero-order terminal layer term $\psi_{0}(x, \tau)$. Since, in view of (3.9), $\widetilde{Q}(x, t) \phi_{0}(x, t)=0$, we derive from Lemma 3.1 that $\phi_{0}(x, t)=\widetilde{\mathbb{1}}(x, t) \beta_{0}(x, t)$. For a suitable function $f(x, t), \dot{f}(x, t)=\frac{\partial f(x, t)}{\partial t}$. Then $\dot{\phi}_{0}(x, t)=\widetilde{\mathbb{1}}(x, t) \dot{\beta}_{0}(x, t)$, so

$$
\widetilde{Q}(x, t) \phi_{1}(x, t)=-\widetilde{\mathbb{1}}(x, t) \dot{\beta}_{0}(x, t)-(\mathcal{L}+\widehat{Q})(x, t)\left(\widetilde{\mathbb{1}}(x, t) \beta_{0}(x, t)\right) \stackrel{\text { def }}{=} \widetilde{b}_{0}(x, t) .
$$

By definition, $\nu(x, t) \widetilde{Q}(x, t)=0$ and $\nu(x, t) \widetilde{\mathbb{1}}=I_{l} \in \mathbb{R}^{l \times l}$, the $l \times l$ identity matrix with $\nu(x, t)$ given in (3.14). Multiplying both sides of equation (3.16) from the left by $\nu(x, t)$, we obtain

$$
\dot{\beta}_{0}(x, t)=-\nu(x, t)(\mathcal{L}+\widehat{Q})(x, t)\left(\widetilde{\mathbb{1}}(x, t) \beta_{0}(x, t)\right) .
$$

In view of (3.12),

$$
\psi_{0}(x, \tau)=\exp (\widetilde{Q}(x, T) \tau)\left(g(x)-\phi_{0}(x, T)\right) .
$$

We demand that $\psi_{0}(x, \tau) \rightarrow 0$ as $\tau \rightarrow \infty$. Letting $\tau \rightarrow \infty$ in (3.18) and noting that $\exp (\widetilde{Q}(x, T) \tau) \rightarrow \bar{P}(x)$ with $\bar{P}(x)$ given in (3.15), we obtain

$$
\bar{P}(x) \psi_{0}(x, 0)=0 .
$$

Multiplying both sides from the left by $\nu(x, T)$, (3.19) is equivalent to $\nu(x, T) \psi_{0}(x, 0)=0$. On the other hand, $\nu(x, T) \psi_{0}(x, 0)=\nu(x, T)\left(g(x)-\phi_{0}(x, T)\right) \nu(x, T) g(x)-\beta_{0}(x, T)$. Thus

$$
\beta_{0}(x, T)=\nu(x, T) g(x) .
$$

Conversely, condition (3.19) holds provided $\beta_{0}(x, T)$ satisfies (3.20). As a result, $\beta_{0}(x, t)$ can be determined from the differential equation (3.17) and the terminal condition (3.20) uniquely. Moreover, with this $\beta_{0}(x, t)$, we also have $\nu(x, t) \widetilde{b}_{0}(x, t)=0$.

3.3. Higher-order terms. Define $Q_{v}(x, t)=\left(\begin{array}{c}\widetilde{Q}(x, t) \\ \nu(x, t)\end{array}\right)$. We have the following lemma.

Lemma 3.4. Under condition (A1), $\operatorname{rank}\left(Q_{v}(x, t)^{\prime} Q_{v}(x, t)\right)=m$.

Proof. Let $w(x, t) \in \mathbb{R}^{m \times 1}$ be a solution of $Q_{v}(x, t) w(x, t)=0$. Then $\widetilde{Q}(x, t) w(x, t)=0$ and $\nu(x, t) w(x, t)=0$. For the first equation above, in view of Lemma 3.1] $w(x, t)=$ $\widetilde{\mathbb{1}}(x, t) \eta(x, t)$. Substituting this into the second equation, we obtain $\nu(x, t) w(x, t)=$ $\nu(x, t) \widetilde{\mathbb{1}}(x, t) \eta(x, t)=\eta(x, t)$. So $\eta(x, t)=0$ and hence $w(x, t) \equiv 0$. Therefore, the only $w(x, t) \in \mathbb{R}^{m \times 1}$ satisfying $w_{1}(x, t) Q_{v}^{1}(x, t)+\cdots+w_{m}(x, t) Q_{v}^{m}(x, t)=0$ is 0 , where $Q_{v}^{k}(x, t)$ is the $k$ th-column of $Q_{v}(x, t)$ for each $k=1, \ldots, m$. Thus the $m$ columns of $Q_{v}(x, t)$ are 
linearly independent. Hence $\operatorname{rank}\left(Q_{v}(x, t)\right)=m$. As a result, $\operatorname{rank}\left(Q_{v}^{\prime}(x, t) Q_{v}(x, t)\right)=$ $\operatorname{rank}\left(Q_{v}(x, t)\right)=m$.

To proceed, for $i>0$, we construct $\phi_{i}(x, t)$ and $\psi_{i}(x, \tau)$ by induction. Suppose that the terms $\phi_{j}(x, t)$ and $\psi_{j}(x, \tau)$ for $j<i$ have been constructed such that $\psi_{j}(x, \tau)$ decays exponentially fast and $\phi_{j}(x, t)$ are smooth. Moreover, assume $\nu(x, t) \widetilde{b}_{j}(x, t)=0$ for all $j<i$. Using (3.9), we have

$$
\widetilde{Q}(x, t) \phi_{i}(x, t)=-\dot{\phi}_{i-1}(x, t)-(\mathcal{L}+\widehat{Q})(x, t) \phi_{i-1}(x, t) \stackrel{\text { def }}{=} \widetilde{b}_{i-1}(x, t) .
$$

Then, by Lemma $3.1 \phi_{i}(x, t)$ is the sum of solutions to the homogeneous equation and a particular solution $\widehat{\phi}_{i}(x, t)$ of the nonhomogeneous equation. It is of the form

$$
\phi_{i}(x, t)=\widetilde{\mathbb{1}}(x, t) \beta_{i}(x, t)+\widehat{\phi}_{i}(x, t) .
$$

Then $\widetilde{Q}(x, t) \widehat{\phi}_{i}(x, t)=\widetilde{b}_{i-1}(x, t)$. The Fredholm alternative leads to $\nu(x, t) \widetilde{b}_{i-1}(x, t)=0$. Denote $Q_{v}(x, t)$ as defined in Lemma 3.4 and $\widehat{b}_{i-1}(x, t)=\left(\begin{array}{c}\widetilde{b}_{i-1}(x, t) \\ 0_{l \times 1}\end{array}\right)$. We can find a unique solution $\widehat{\phi}_{i}(x, t)$ of (3.21) such that $\widetilde{Q}(x, t) \widehat{\phi}_{i}(x, t)=\widetilde{b}_{i-1}(x, t)$ and $\nu(x, t)$ is orthogonal to $\widehat{\phi}_{i}(x, t)$. That is, $Q_{v}(x, t) \widehat{\phi}_{i}(x, t)=\widehat{b}_{i-1}(x, t)$. Lemma 3.4 implies that the particular solution is uniquely determined by

$$
\widehat{\phi}_{i}(x, t)=\left(Q_{v}(x, t)^{\prime} Q_{v}(x, t)\right)^{-1} Q_{v}(x, t)^{\prime} \widehat{b}_{i-1}(x, t) .
$$

On the other hand, $\widetilde{Q}(x, t) \phi_{i+1}(x, t)=-\dot{\phi}_{i}(x, t)-(\mathcal{L}+\widehat{Q})(x, t) \phi_{i}(x, t) \stackrel{\text { def }}{=} \widetilde{b}_{i}(x, t)$. Multiplying both sides by $\nu(x, t)$ from the left and noting (3.22), we deduce

$\dot{\beta}_{i}(x, t)=-\nu(x, t) \dot{\widehat{\phi}}_{i}(x, t)-\nu(x, t)(\mathcal{L}+\widehat{Q})(x, t) \widehat{\phi}_{i}(x, t)-\nu(x, t)(\mathcal{L}+\widehat{Q})(x, t)\left(\widetilde{\mathbb{1}}(x, t) \beta_{i}(x, t)\right)$.

Equation (3.24) is uniquely solvable if the terminal condition is specified. We need to use the terminal layer term to determine the terminal condition. In view of (3.12),

$$
\psi_{i}(x, \tau)=-\exp (\widetilde{Q}(x, T) \tau) \phi_{i}(x, T)+\int_{0}^{\tau} \exp (\widetilde{Q}(x, T)(\tau-s)) r_{i}(x, s) d s .
$$

We demand that $\psi_{i}(x, \tau) \rightarrow 0$ as $\tau \rightarrow \infty$. Letting $\tau \rightarrow \infty$ in 3.25 and noting that $\exp (\widetilde{Q}(x, T) \tau) \rightarrow \bar{P}(x)$ with $\bar{P}(x)$ given in (3.15) and that $r_{i}(x, t)$ decays exponentially fast, we obtain

$$
\bar{P}(x) \psi_{i}(x, 0)+\int_{0}^{\infty} \bar{P}(x) r_{i}(x, s) d s=0 .
$$

By multiplying both sides from the left by $\nu(x, T)$, the above equation is equivalent to

$$
\nu(x, T) \psi_{i}(x, 0)+\int_{0}^{\infty} \nu(x, T) r_{i}(x, s) d s=0 .
$$

We have

$$
\begin{aligned}
& \nu(x, T) \psi_{i}(x, 0)+\int_{0}^{\infty} \nu(x, T) r_{i}(x, s) d s=-\nu(x, T) \phi_{i}(x, T)+\int_{0}^{\infty} \nu(x, T) r_{i}(x, s) d s \\
& =-\beta_{i}(x, T)-\nu(x, T) \widehat{\phi}_{i}(x, T)+\int_{0}^{\infty} \nu(x, T) r_{i}(s) d s=-\beta_{i}(x, T)+\int_{0}^{\infty} \nu(x, T) r_{i}(x, s) d s .
\end{aligned}
$$


Note that the integral involving $r_{i}(x, s)$ is well defined since $\left|r_{i}(x, s)\right| \leq C \mathrm{e}^{-\gamma s}$ by the induction hypothesis. By virtue of (3.26) and (3.28), we obtain

$$
\beta_{i}(x, T)=\int_{0}^{\infty} \nu(x, T) r_{i}(x, s) d s .
$$

Conversely, when $\beta_{i}(x, T)$ satisfies (3.24), condition (3.26) holds as desired. Then

$$
\phi_{i}(x, t)=\widetilde{\mathbb{1}}(x, t) \beta_{i}(x, t)+\widehat{\phi}_{i}(x, t)=\widetilde{\mathbb{1}}(x, t) \beta_{i}(x, t)+\left(Q_{v}(x, t)^{\prime} Q_{v}(x, t)\right)^{-1} Q_{v}(x, t)^{\prime} \widehat{b}_{i-1}
$$

with $\beta_{i}(x, t)$ uniquely determined by the differential equations (3.24) and the terminal condition (3.29). In addition, $\nu(x, t) \widetilde{b}_{i}(x, t)=0$. Moreover, by the construction, it is readily seen that $\psi_{i}(x, \tau)$ decays exponentially fast.

Proposition 3.5. $\phi_{i} \in C^{2(n+2-i), n+2-i}([0,1] \times[0, T])$ for any $i=0, \ldots, n+2$.

Proof. We prove this by induction. First, denote $Q_{a}(x, t)=(\widetilde{Q}(x, t) \widetilde{\mathbb{1}}(x, t))$. Then $\nu(x, t) Q_{a}=\left(0_{l \times m} I_{l}\right)$. Moreover, using irreducibility of $\widetilde{Q}^{\imath}(x, t)$ for $\imath=1, \ldots, l$, we can follow the proof of Lemma 3.4 to prove that $\operatorname{rank}\left(Q_{a}^{\prime}(x, t) Q_{a}(x, t)\right)=m$. So $\nu(x, t)=\left(0_{l \times m} I_{l}\right) Q_{a}^{\prime}(x, t)\left(Q_{a}(x, t) Q_{a}^{\prime}(x, t)\right)^{-1}$. Thus $\nu(\cdot, \cdot) \in C^{2(n+2), n+2}([0,1] \times[0, T])$ and $\widetilde{\mathbb{1}}(\cdot, \cdot) \in C^{2(n+2), n+2}([0,1] \times[0, T])$. So (3.17) implies that $\beta_{0}(\cdot, \cdot) \in C^{2(n+2), n+2}([0,1]$ $\times[0, T])$. So $\phi_{0} \in C^{2(n+2), n+2}([0,1] \times[0, T])$. Assume that $\phi_{j} \in C^{2(n+2-j), n+2-j}([0,1] \times$ $[0, T])$ for any $j<i$. In view of (3.21), we deduce $\widehat{b}_{i-1} \in C^{2(n+2-i), n+2-i}([0,1] \times[0, T])$. Then we derive from (3.23) and (3.24) that $\widehat{\phi}_{i} \in C^{2(n+2-i), n+2-i}([0,1] \times[0, T])$ and $\beta_{i} \in C^{2(n+2-i), n+2-i}([0,1] \times[0, T])$. Thus (3.30) implies $\phi_{i} \in C^{2(n+2-i), n+2-i}([0,1] \times$ $[0, T])$.

Lemma 3.6. For a fixed integer $i$ and an integer $h$ satisfying $0 \leq h \leq 2(n+2-i)$, put $w_{i}^{h}(x, \tau)=\frac{\partial^{h} \psi_{i}(x, \tau)}{\partial x^{h}}$. Assume for any $\tau$ and $x, \max _{h=0, \ldots, 2(n+2-i)}\left|\frac{\partial^{h} r_{i}(x, \tau)}{\partial x^{h}}\right| \leq C \mathrm{e}^{-\gamma \tau}$ and $\left|\psi_{i}(x, \tau)\right| \leq C \mathrm{e}^{-\gamma \tau}$. Then for any $\tau, x, \max _{h=0, \ldots, 2(n+2-i)}\left|w_{i}^{h}(x, \tau)\right| \leq C \mathrm{e}^{-\gamma \tau}$.

Proof. First, $\left|w_{i}^{0}(x, \tau)\right|=\left|\psi_{i}(x, \tau)\right| \leq C \mathrm{e}^{-\gamma \tau}$. Suppose for any $\widetilde{h}<h,\left|w_{i}^{\widetilde{h}}(x, \tau)\right| \leq$ $C \mathrm{e}^{-\gamma \tau}$. Then (3.11) implies

$$
\begin{aligned}
\frac{\partial w_{i}^{h}(x, \tau)}{\partial \tau} & =\widetilde{Q}(x, T) w_{i}^{h}(x, \tau)+\sum_{\widetilde{h}=0}^{h-1}\left(\begin{array}{l}
h \\
\widetilde{h}
\end{array}\right) \frac{\partial^{h-\widetilde{h}} \widetilde{Q}(x, T)}{\partial x^{h-\widetilde{h}}} w_{i}^{\widetilde{h}}(x, \tau)+\frac{\partial^{h} r_{i}(x, \tau)}{\partial x^{h}} \\
w_{i}^{h}(x, 0) & =\frac{\partial^{h} \psi_{i}(x, 0)}{\partial x^{h}}
\end{aligned}
$$

It follows that

$$
\begin{aligned}
w_{i}^{h}(x, \tau)= & \exp (\widetilde{Q}(x, T) \tau) w_{i}^{h}(x, 0)+\int_{0}^{\tau} \exp (\widetilde{Q}(x, T)(\tau-s)) \frac{\partial^{h} r_{i}(x, s)}{\partial x^{h}} d s \\
& +\sum_{\widetilde{h}=0}^{h-1} \int_{0}^{\tau}\left(\begin{array}{l}
h \\
\widetilde{h}
\end{array}\right) \exp (\widetilde{Q}(x, T)(\tau-s)) \frac{\partial^{h-\widetilde{h}} \widetilde{Q}(x, T)}{\partial x^{h-\widetilde{h}}} w_{i}^{\widetilde{h}}(x, s) d s .
\end{aligned}
$$


Define

$$
\begin{aligned}
\widetilde{w}_{i}^{h}(x) \stackrel{\text { def }}{=} \nu(x, & T) w_{i}^{h}(x, 0)+\int_{0}^{\infty} \nu(x, T) \frac{\partial^{h} r_{i}(x, s)}{\partial x^{h}} d s \\
& +\sum_{\widetilde{h}=0}^{h-1} \int_{0}^{\infty}\left(\begin{array}{c}
h \\
\widetilde{h}
\end{array}\right) \nu(x, T) \frac{\partial^{h-\widetilde{h}} \widetilde{Q}(x, T)}{\partial x^{h-\widetilde{h}}} w_{i}^{\widetilde{h}}(x, s) d s .
\end{aligned}
$$

We claim that $\widetilde{w}_{i}^{h}(x)=0$. Note that 3.32 implies that

$$
\begin{aligned}
& \left|w_{i}^{h}(x, \tau)\right| \leq|\exp (\widetilde{Q}(x, T) \tau)-\bar{P}(x)|\left|w_{i}^{h}(x, 0)\right| \\
& \quad+\sum_{\widetilde{h}=0}^{h-1} \int_{\tau}^{\infty}\left(\begin{array}{c}
h \\
\widetilde{h}
\end{array}\right)|\bar{P}(x)|\left|\frac{\partial^{h-\widetilde{h}} \widetilde{Q}(x, T)}{\partial x^{h-\widetilde{h}}}\right|\left|w_{i}^{\widetilde{h}}(x, s)\right| d s \\
& \quad+\sum_{\widetilde{h}=0}^{h-1} \int_{0}^{\tau}\left(\begin{array}{c}
h \\
\widetilde{h}
\end{array}\right)|\exp (\widetilde{Q}(x, T)(\tau-s))-\bar{P}(x)|\left|\frac{\partial^{h-\widetilde{h}} \widetilde{Q}(x, T)}{\partial x^{h-\widetilde{h}}}\right|\left|w_{i}^{\widetilde{h}}(x, s)\right| d s \\
& \quad+\int_{0}^{\tau}|\exp (\widetilde{Q}(x, T)(\tau-s))-\bar{P}(x)|\left|\frac{\partial^{h} r_{i}(x, s)}{\partial x^{h}}\right| d s+\int_{\tau}^{\infty}|\bar{P}(x)|\left|\frac{\partial^{h} r_{i}(x, s)}{\partial x^{h}}\right| d s \\
& \leq C \mathrm{e}^{-\gamma \tau}+(h+1) \int_{0}^{\tau} C \mathrm{e}^{-\gamma(\tau-s)} \mathrm{e}^{-\gamma s} d s+(h+1) \int_{\tau}^{\infty} C \mathrm{e}^{-\gamma s} d s=C \mathrm{e}^{-\gamma \tau} .
\end{aligned}
$$

Note that we use $\gamma$ to represent a generic positive constant, whose value may be different for different appearances. Now we prove the above claim. In fact, 3.27) implies for any $\widetilde{h}$,

$$
\begin{aligned}
& \sum_{\iota=0}^{h}\left(\begin{array}{l}
h \\
\iota
\end{array}\right) \frac{\partial^{h-\iota} \nu(x, T)}{\partial x^{h-\iota}} w_{i}^{\iota}(x, 0)+\sum_{\iota=0}^{h} \int_{0}^{\infty}\left(\begin{array}{l}
h \\
\iota
\end{array}\right) \frac{\partial^{h-\iota} \nu(x, T)}{\partial x^{h-\iota}} \frac{\partial^{\iota} r_{i}(x, s)}{\partial x^{\iota}} d s=0, \\
& \sum_{\iota=0}^{h}\left(\begin{array}{l}
\widetilde{h} \\
\iota
\end{array}\right) \frac{\partial^{\widetilde{h}-\iota} \nu(x, T)}{\partial x^{\widetilde{h}-\iota}} \frac{\partial^{\iota} \widetilde{Q}(x, T)}{\partial x^{\iota}}=0 .
\end{aligned}
$$

We derive from (3.11), (3.33), and (3.31) that

$$
\begin{aligned}
\widetilde{w}_{i}^{h}(x)= & -\sum_{\iota=0}^{h-1}\left(\begin{array}{c}
h \\
\iota
\end{array}\right) \frac{\partial^{h-\iota} \nu}{\partial x^{h-\iota}}(x, T) w_{i}^{\iota}(x, 0)-\sum_{\iota=0}^{h-1} \int_{0}^{\infty}\left(\begin{array}{c}
h \\
\iota
\end{array}\right) \frac{\partial^{h-\iota} \nu(x, T)}{\partial x^{h-\iota}} \frac{\partial^{\iota} r_{i}(x, s)}{\partial x^{\iota}} d s \\
& -\sum_{\widetilde{h}=0}^{h-1} \int_{0}^{\infty}\left(\begin{array}{c}
h \\
\tilde{h}
\end{array}\right) \sum_{\iota=0}^{h-\widetilde{h}-1}\left(\begin{array}{c}
h-\widetilde{h} \\
\iota
\end{array}\right) \frac{\partial^{h-\widetilde{h}-\iota} \nu(x, T)}{\partial x^{h-\widetilde{h}-\iota}} \frac{\partial^{\iota} \widetilde{Q}(x, T)}{\partial x^{\iota}} w_{i}^{\widetilde{h}}(x, s) d s .
\end{aligned}
$$

On the other hand, the last term of the above equation equals

$$
\begin{aligned}
& -\sum_{\widetilde{h}=0}^{h-1} \int_{0}^{\infty} \sum_{\iota=1}^{h-\widetilde{h}} \frac{h !}{\widetilde{h} ! \iota !(h-\widetilde{h}-\iota) !} \frac{\partial^{\iota} \nu(x, T)}{\partial x^{\iota}} \frac{\partial^{h-\widetilde{h}-\iota} \widetilde{Q}(x, T)}{\partial x^{h-\widetilde{h}-\iota}} w_{i}^{\widetilde{h}}(x, s) d s \\
& =-\sum_{\iota=1}^{h} \int_{0}^{\infty} \sum_{\widetilde{h}=0}^{h-\iota}\left(\begin{array}{c}
h \\
h-\iota
\end{array}\right)\left(\begin{array}{c}
h-\iota \\
\widetilde{h}
\end{array}\right) \frac{\partial^{\iota} \nu(x, T)}{\partial x^{\iota}} \frac{\partial^{h-\widetilde{h}-\iota} \widetilde{Q}(x, T)}{\partial x^{h-\widetilde{h}-\iota}} w_{i}^{\widetilde{h}}(x, s) d s \\
& =-\sum_{\iota=0}^{h-1}\left(\begin{array}{c}
h \\
\iota
\end{array}\right) \int_{0}^{\infty} \sum_{\widetilde{h}=0}^{\iota}\left(\begin{array}{c}
\iota \\
\widetilde{h}
\end{array}\right) \frac{\partial^{h-\iota} \nu(x, T)}{\partial x^{h-\iota}} \frac{\partial^{\iota-\widetilde{h}} \widetilde{Q}(x, T)}{\partial x^{\iota-\widetilde{h}}} w_{i}^{\widetilde{h}}(x, s) d s .
\end{aligned}
$$


Hence,

$$
\begin{aligned}
\widetilde{w}_{i}^{h}(x)= & -\sum_{\iota=0}^{h-1}\left(\begin{array}{l}
h \\
\iota
\end{array}\right) \frac{\partial^{h-\iota} \nu(x, T)}{\partial x^{h-\iota}} w_{i}^{\iota}(x, 0) \\
& -\sum_{\iota=0}^{h-1}\left(\begin{array}{l}
h \\
\iota
\end{array}\right) \frac{\partial^{h-\iota} \nu(x, T)}{\partial x^{h-\iota}} \int_{0}^{\infty}\left(\sum_{\widetilde{h}=0}^{\iota}\left(\begin{array}{c}
\iota \\
h
\end{array}\right) \frac{\partial^{\iota-\widetilde{h}} \widetilde{Q}(x, T)}{\partial x^{\iota-\widetilde{h}}} w_{i}^{\widetilde{h}}(x, s)+\frac{\partial^{\iota} r}{\partial x^{\iota} i}(x, s)\right) d s \\
= & -\sum_{\iota=0}^{h-1}\left(\begin{array}{l}
h \\
\iota
\end{array}\right) \frac{\partial^{h-\iota} \nu}{\partial x^{h-\iota}}(x, T) w_{i}^{\iota}(x, 0)-\sum_{\iota=0}^{h-1}\left(\begin{array}{l}
h \\
\iota
\end{array}\right) \frac{\partial^{h-\iota} \nu(x, T)}{\partial x^{h-\iota}} \int_{0}^{\infty} \frac{\partial w_{i}^{\iota}(x, s)}{\partial s} d s \\
= & -\sum_{\iota=0}^{h-1}\left(\begin{array}{l}
h \\
\iota
\end{array}\right) \frac{\partial^{h-\iota} \nu(x, T)}{\partial x^{h-\iota}} \lim _{s \rightarrow \infty} w_{i}^{\iota}(x, s)=0 .
\end{aligned}
$$

Proposition 3.7. There exist constants $C$ and $0<\gamma_{i}<\gamma$ such that for any $0 \leq i \leq n+1$,

$$
\max _{h=0, \ldots, 2(n+2-i)}\left|\frac{\partial^{h} \psi_{i}(x, \tau)}{\partial x^{h}}\right| \leq C \mathrm{e}^{-\gamma_{i} \tau}, \quad \forall \tau \geq 0,0 \leq x \leq 1 .
$$

Proof. First, under condition (3.19), we have

$$
\left|\psi_{0}(x, \tau)\right|=\left|\bar{P}(x) \psi_{0}(x, 0)+(\exp (\widetilde{Q}(x, T) \tau)-\bar{P}(x)) \psi_{0}(x, 0)\right| \leq C \varepsilon^{-\gamma \tau} .
$$

Applying Lemma 3.6 with $r_{0}=0$, we deduce that (3.34) is valid for $i=0$. Assume that for any $j<i, \max _{h=0, \ldots, 2 n+2}\left|\frac{\partial^{h} \psi_{j}(x, \tau)}{\partial x^{h}}\right| \leq C \varepsilon^{-\gamma_{j} \tau}$. Then $\max _{h=0, \ldots, 2 n+2}\left|\frac{\partial^{h} r_{i}(x, \tau)}{\partial x^{h}}\right| \leq C \varepsilon^{-\gamma \tau}$, with $\gamma=\min \left(\gamma_{1}, \ldots, \gamma_{i-1}\right)$. Under condition (3.26), we deduce

$$
\begin{aligned}
\left|\psi_{i}(x, \tau)\right| \leq\left|(\exp (\widetilde{Q}(x, T) \tau)-\bar{P}) \psi_{i}(x, 0)\right|+\left|\int_{\tau}^{\infty}-\bar{P} r_{i}(x, s) d s\right| \\
\quad+\left|\int_{0}^{\tau}(\exp (\widetilde{Q}(x, T)(\tau-s))-\bar{P}) r_{i}(x, s) d s\right| \\
\leq C \varepsilon^{-\gamma \tau}+C \int_{0}^{\tau} \varepsilon^{-\gamma(\tau-s)} \varepsilon^{-\gamma s} d s+C \int_{\tau}^{\infty} \varepsilon^{-\gamma s} d s \leq C \mathrm{e}^{-\gamma \tau} .
\end{aligned}
$$

Lemma 3.6 again shows that (3.34) holds for $i$. This completes the proof by induction.

3.4. Error estimates. For a suitable function $f$, define $\mathbf{L}^{\varepsilon} f=\frac{\partial f}{\partial t}+Q^{\varepsilon} f+\mathcal{L} f$.

Lemma 3.8. Let $\omega(x, s)$ be the solution of the following equation:

$$
\begin{aligned}
& \mathbf{L}^{\varepsilon}(x, t) \omega(x, t)=\zeta(x, t), \quad \text { for } t<T, \\
& \omega(x, T)=0 .
\end{aligned}
$$

Then $\omega_{i}(x, t)=-E \int_{t}^{T} \zeta\left(Y^{\varepsilon, x, i}(s)\right) d s$, where $Y^{\varepsilon, x, i}(t)=\left(X^{\varepsilon}(t), \alpha^{\varepsilon}(t)\right)$ satisfies $X^{\varepsilon}(T)=$ $x$ and $\alpha^{\varepsilon}(T)=i \in \mathcal{M}$.

Proof. Since $\mathbf{L}^{\varepsilon}$ is the generator of $Y^{\varepsilon}$, by virtue of Itô's formula, $\omega(x, t)=\omega\left(Y^{\varepsilon}(T)\right)-\omega\left(Y^{\varepsilon}(t)\right)=-\int_{t}^{T} \mathbf{L}^{\varepsilon} \omega\left(Y^{\varepsilon}(s)\right) d s+M(t)=-\int_{t}^{T} \zeta\left(Y^{\varepsilon}(s)\right) d s+M(t)$, 
where $M(t)$ is a martingale. Taking expectation in (3.36) leads to the desired assertion.

Lemma 3.9. Suppose that $\zeta \in C([0,1] \times[0, T])$ is periodic in $x \in[0,1]$, satisfying $\sup _{(x, t) \in[0,1] \times[0, T]}|\zeta(x, t)| \leq C \varepsilon^{\kappa}$. Let $\xi^{\varepsilon}(x, t)$ be a solution to

$$
\mathbf{L}^{\varepsilon} \xi^{\varepsilon}(x, t)=\zeta(x, t), \quad \xi^{\varepsilon}(x, T)=0, \quad \forall x \in[0,1]
$$

Then $\sup _{(x, t) \in[0,1] \times[0, T]}\left|\xi^{\varepsilon}(x, t)\right| \leq C \varepsilon^{\kappa}$.

Proof. The desired result follows from the previous lemma.

Theorem 3.10. There exists a $C>0$ such that

$$
\sup _{(x, t) \in[0,1] \times[0, T]}\left|p^{\varepsilon}(x, t)-\Phi_{n}^{\varepsilon}(x, t)-\Psi_{n}^{\varepsilon}(x, \tau)\right| \leq C \varepsilon^{n+1} .
$$

Proof. Using $\tau$ defined in (3.7), put $e^{\varepsilon, \kappa}(x, t)=p^{\varepsilon}(x, t)-\Phi_{\kappa}^{\varepsilon}(x, t)-\Psi_{\kappa}^{\varepsilon}(x, \tau)$. Then $\mathbf{L}^{\varepsilon} u^{\varepsilon}(x, t)=0$ and $\mathbf{L}^{\varepsilon} e^{\varepsilon, \kappa}(x, t)=-\mathbf{L}^{\varepsilon} \Phi_{\kappa}^{\varepsilon}(x, t)-\mathbf{L}^{\varepsilon} \Psi_{\kappa}^{\varepsilon}(x, \tau)$. Moreover

$$
\begin{aligned}
\mathbf{L}^{\varepsilon} \Phi_{\kappa}^{\varepsilon}(x, t) & =\sum_{i=0}^{\kappa} \varepsilon^{i} \dot{\phi}_{i}(x, t)+\sum_{i=0}^{\kappa} \varepsilon^{i-1} \widetilde{Q}(x, t) \phi_{i}(x, t)+\sum_{i=0}^{\kappa} \varepsilon^{i}(\mathcal{L}+\widehat{Q})(x, t) \phi_{i}(x, t) \\
& =-\varepsilon^{\kappa} \widetilde{Q}(x, t) \phi_{\kappa+1}(x, t)+\underbrace{\varepsilon^{-1} \widetilde{Q}(x, t) \phi_{0}(x, t)}_{0}
\end{aligned}
$$

So $\left|\mathbf{L}^{\varepsilon} \Phi_{\kappa}^{\varepsilon}(x, t)\right| \leq C \varepsilon^{\kappa}$. Note that $\varepsilon \frac{d}{d t} \psi_{i}\left(\frac{T-t}{\varepsilon}\right)=-\frac{d}{d \tau} \psi_{i}(x, \tau)$, which yields

$$
\begin{aligned}
& \mathbf{L}^{\varepsilon} \Psi_{\kappa}^{\varepsilon}(x, \tau)=\sum_{i=0}^{\kappa}-\varepsilon^{i-1} \frac{\partial \psi_{i}(x, \tau)}{\partial \tau}+\sum_{i=0}^{\kappa} \varepsilon^{i-1} \widetilde{Q}(x, t) \psi_{i}(x, \tau)+\sum_{i=0}^{\kappa} \varepsilon^{i}(\mathcal{L}+\widehat{Q})(x, t) \psi_{i}(x, \tau) \\
& =\sum_{i=0}^{\kappa} \varepsilon^{i-1}\left(-\widetilde{Q}(x, T) \psi_{i}(x, \tau)-r_{i}(x, \tau)\right)+\sum_{i=0}^{\kappa} \varepsilon^{i-1} \widetilde{Q}(x, t) \psi_{i}(x, \tau) \\
& \quad+\sum_{i=0}^{\kappa} \varepsilon^{i}(\mathcal{L}+\widehat{Q})(x, t) \psi_{i}(x, \tau) \\
& =\sum_{i=0}^{\kappa} \varepsilon^{i-1}(-\widetilde{Q}(x, T)+\widetilde{Q}(x, t)) \psi_{i}(x, \tau)-\sum_{i=0}^{\kappa} \varepsilon^{i-1} r_{i}(x, \tau)+\sum_{i=0}^{\kappa} \varepsilon^{i}(\mathcal{L}+\widehat{Q})(x, t) \psi_{i}(x, \tau)
\end{aligned}
$$


For the second term, we have

$$
\begin{aligned}
& \sum_{i=0}^{\kappa} \varepsilon^{i-1} r_{i}(x, \tau)=\sum_{i=1}^{\kappa} \varepsilon^{i-1} \sum_{j=0}^{i-1}\left((-\tau)^{i-j} \frac{\widetilde{Q}^{(i-j)}(x, T)}{(i-j) !}\right. \\
& \left.\quad+(-\tau)^{i-j-1} \frac{(\mathcal{L}+\widehat{Q})^{(i-j-1)}(x, T)}{(i-j-1) !}\right) \psi_{j}(x, \tau) \\
& =\sum_{j=0}^{\kappa-1} \sum_{i=j+1}^{\kappa}\left(\varepsilon^{i-1}(-\tau)^{i-j} \frac{\widetilde{Q}^{(i-j)}(x, T)}{(i-j) !}+\varepsilon^{i-1}(-\tau)^{i-j-1} \frac{(\mathcal{L}+\widehat{Q})^{(i-j-1)}(x, T)}{(i-j-1) !}\right) \psi_{j}(x, \tau) \\
& =\sum_{j=0}^{\kappa-1} \varepsilon^{j-1}\left(\sum_{i=1}^{\kappa-j}(t-T)^{i} \frac{\widetilde{Q}^{(i)}(x, T)}{i !}\right) \psi_{j}(x, \tau) \\
& \quad+\sum_{j=0}^{\kappa-1} \varepsilon^{j}\left(\sum_{i=0}^{\kappa-j-1}(t-T)^{i} \frac{(\mathcal{L}+\widehat{Q})^{(i)}(x, T)}{i !}\right) \psi_{j}(x, \tau) .
\end{aligned}
$$

Therefore,

$$
\begin{aligned}
\mathbf{L}^{\varepsilon} \Psi_{\kappa}^{\varepsilon}(x, \tau)= & \varepsilon^{\kappa-1}(-\widetilde{Q}(x, T)+\widetilde{Q}(x, t)) \psi_{\kappa}(x, \tau)+\varepsilon^{\kappa}(\mathcal{L}+\widehat{Q})(x, t) \psi_{\kappa}(x, \tau) \\
& +\sum_{j=0}^{\kappa-1} \varepsilon^{j-1}\left(\widetilde{Q}(x, t)-\sum_{i=0}^{\kappa-j}(t-T)^{i} \frac{\widetilde{Q}^{(i)}(x, T)}{i !}\right) \psi_{j}(x, \tau) \\
& +\sum_{j=0}^{\kappa-1} \varepsilon^{j}\left((\mathcal{L}+\widehat{Q})(x, t)-\sum_{i=0}^{\kappa-j-1}(t-T)^{i} \frac{(\mathcal{L}+\widehat{Q})^{(i)}(x, T)}{i !}\right) \psi_{j}(x, \tau) .
\end{aligned}
$$

Using Taylor expressions and Proposition 3.7, we obtain

$$
\begin{aligned}
\left|\mathbf{L}^{\varepsilon} \Psi_{\kappa}^{\varepsilon}(x, \tau)\right| \leq & C \varepsilon^{\kappa-1}|t-T| \mathrm{e}^{-\gamma \tau}+C \varepsilon^{\kappa}+C \sum_{j=0}^{\kappa-1} \varepsilon^{j-1}|t-T|^{\kappa-j+1} \mathrm{e}^{-\gamma \tau} \\
& +C \sum_{j=0}^{\kappa-1} \varepsilon^{j}|t-T|^{\kappa-j} \mathrm{e}^{-\gamma \tau} \\
= & C \varepsilon^{\kappa} \tau \mathrm{e}^{-\gamma \tau}+C \varepsilon^{\kappa}+C \sum_{j=0}^{\kappa-1} \varepsilon^{\kappa} \tau^{\kappa-j+1} \mathrm{e}^{-\gamma \tau}+C \sum_{j=0}^{\kappa-1} \varepsilon^{\kappa} \tau^{\kappa-j} \mathrm{e}^{-\gamma \tau} \leq C \varepsilon^{\kappa} .
\end{aligned}
$$

This together with the estimates on $\mathbf{L}^{\varepsilon} \Phi_{\kappa}(x, t)$ yields $\sup _{(x, t) \in[0,1] \times[0, T]}\left|\mathbf{L}^{\varepsilon} e^{\varepsilon, \kappa}(x, t)\right| \leq$ $C \varepsilon^{\kappa}$. Noting $e^{\varepsilon, \kappa}(x, T)=0$, Lemma 3.9 implies $\sup _{(x, t) \in[0,1] \times[0, T]}\left|e^{\varepsilon, \kappa}(x, t)\right| \leq C \varepsilon^{\kappa}$. Taking $\kappa=n+1$, we obtain $\sup _{(x, t) \in[0,1] \times[0, T]}\left|e^{\varepsilon, n+1}(x, t)\right|=O\left(\varepsilon^{n+1}\right)$. Finally, note that

$$
e^{\varepsilon, n+1}(x, t)=e^{\varepsilon, n}(x, t)+\varepsilon^{n+1} \phi_{n+1}(x, t)+\varepsilon^{n+1} \psi_{n+1}(x, \tau) .
$$

The continuity of $\phi_{n+1}(x, t)$ and the exponential decay properties of $\psi_{n+1}(x, \tau)$ yield that

$$
\sup _{(x, t) \in[0,1] \times[0, T]}\left|\varepsilon^{n+1} \phi_{n+1}(x, t)+\varepsilon^{n+1} \psi_{n+1}(x, \tau)\right| \leq C \varepsilon^{n+1} .
$$

Substituting this into (3.39), we obtain $\sup _{(x, t) \in[0,1] \times[0, T]}\left|e^{\varepsilon, n}(x, t)\right| \leq C \varepsilon^{n+1}$. 
4. Fast diffusion. Suppose that $\alpha(t)$ is a jump process with generator $Q(x, t)$. Let two operators $\widetilde{\mathcal{L}}$ and $\widehat{\mathcal{L}}$ be defined similarly to (2.3) as

$$
\begin{aligned}
\widetilde{\mathcal{L}}(x, t) u(x, k, t) & =\frac{1}{2} \widetilde{a}(x, k, t) \frac{\partial^{2}}{\partial x^{2}} u(x, k, t)+\widetilde{b}(x, k, t) \frac{\partial}{\partial x} u(x, k, t), \\
\widehat{\mathcal{L}}(x, t) u(x, k, t) & =\frac{1}{2} \widehat{a}(x, k, t) \frac{\partial^{2}}{\partial x^{2}} u(x, k, t)+\widehat{b}(x, k, t) \frac{\partial}{\partial x} u(x, k, t) .
\end{aligned}
$$

Let $\mathcal{L}^{\varepsilon}(x, t)=\frac{\widetilde{\mathcal{L}}(x, t)}{\varepsilon}+\widehat{\mathcal{L}}(x, t)$. Throughout this section, in addition to assumptions (A4), we also assume the following conditions.

(A6) $\widetilde{a}(x, k, t)>0$ for all $x, t$ and $k$. Changing part of the diffusion is uniformly elliptic.

(A7) For each $k \in \mathcal{M}$,

- $\widetilde{a}(\cdot, k, t), \widehat{a}(\cdot, k, t), \widetilde{b}(\cdot, k, t), \widehat{b}(\cdot, k, t)$ are periodic in $x$ with period 1 for each $t \in[0, T]$ and $\widetilde{a}(\cdot, k, \cdot), \widehat{a}(\cdot, k, \cdot), \widetilde{b}(\cdot, k, \cdot), \widehat{b}(\cdot, k, \cdot) \in C^{2(n+2), n+2}([0,1] \times[0, T])$.

- $\widetilde{a}(\cdot, k, T) \in C^{2 n+6}([0,1])$ and $\widetilde{b}(\cdot, k, T) \in C^{2 n+5}([0,1])$.

(A8) $Q(\cdot, \cdot) \in C^{2(n+2), n+2}([0,1] \times[0, T])$.

We consider

$$
-\frac{\partial \widetilde{u}^{\varepsilon}}{\partial t}=Q(x, t) \widetilde{u}^{\varepsilon}+\mathcal{L}^{\varepsilon}(x, t) \widetilde{u}^{\varepsilon}, \quad \widetilde{u}^{\varepsilon}(x, T)=g(x) .
$$

Similarly to Section 3. we seek asymptotic expansions of the form (3.8). Substituting the expansions in (4.40), we obtain

$$
\begin{aligned}
& \widetilde{\mathcal{L}}(x, t) \phi_{0}(x, t)=0, \\
& \widetilde{\mathcal{L}}(x, t) \phi_{1}(x, t)=-\frac{\partial}{\partial t} \phi_{0}(x, t)-(\widehat{\mathcal{L}}+Q)(x, t) \phi_{0}(x, t) \stackrel{\text { def }}{=} \varsigma_{0}(x, t), \\
& \ldots \ldots \\
& \widetilde{\mathcal{L}}(x, t) \phi_{i+1}(x, t)=-\frac{\partial}{\partial t} \phi_{i}(x, t)-(\widehat{\mathcal{L}}+Q)(x, t) \phi_{i}(x, t) \stackrel{\text { def }}{=} \varsigma_{i}(x, t),
\end{aligned}
$$

where $i=2, \ldots, n+2$. Likewise, substituting $\Psi_{\kappa}(x, \tau)$ for $\kappa \leq n+2$ into (4.40) and applying Taylor expansions for $\widetilde{\mathcal{L}}(x, T-\varepsilon \tau), \widehat{\mathcal{L}}(x, T-\varepsilon \tau)$ and $Q(x, T-\varepsilon \tau)$, we arrive at

$$
\begin{aligned}
& \frac{\partial \psi_{0}(x, \tau)}{\partial \tau}=\widetilde{\mathcal{L}}(x, T) \psi_{0}(x, \tau), \\
& \frac{\partial \psi_{1}(x, \tau)}{\partial \tau}=\widetilde{\mathcal{L}}(x, T) \psi_{1}(x, \tau)+\left(-\tau \widetilde{\mathcal{L}}^{(1)}(x, T)+(\widehat{\mathcal{L}}+Q)(x, T)\right) \psi_{0}(x, \tau), \\
& \cdots \cdots \\
& \frac{\partial \psi_{i}(x, \tau)}{\partial \tau}=\widetilde{\mathcal{L}}(x, T) \psi_{i}(x, \tau)+r_{i}(x, \tau), \\
& r_{i}(x, \tau)=\sum_{j=0}^{i-1}\left((-\tau)^{i-j} \frac{\widetilde{\mathcal{L}}^{(i-j)}(x, T)}{(i-j) !}+(-\tau)^{i-j-1} \frac{(\widehat{\mathcal{L}}+Q)^{(i-j-1)}(x, T)}{(i-j-1) !}\right) \psi_{j}(x, \tau),
\end{aligned}
$$

where $\widetilde{\mathcal{L}}^{(i)}(x, T)=\frac{\partial^{i} \widetilde{\mathcal{L}}(x, T)}{\partial t^{i}}, \quad \widehat{\mathcal{L}}^{(i)}(x, T)=\frac{\partial^{i} \widehat{\mathcal{L}}(x, T)}{\partial t^{i}}, \quad Q^{(i)}(x, T)=\frac{\partial^{i} Q(x, T)}{\partial t^{i}}$.

From the initial condition, we derive $\phi_{0}(x, T)+\psi_{0}(x, 0)=g(x)$ and $\phi_{i}(x, T)+\psi_{i}(x, 0)$ $=0$, for $i>0$. We recall that the adjoint operator of $\widetilde{\mathcal{L}}_{k}$ has the form

$$
\widetilde{\mathcal{L}}_{k}^{*}(x, t) u(x, k, t)=\frac{\partial^{2}}{\partial x^{2}}\left[\frac{1}{2} \widetilde{a}(x, k, t) u(x, k, t)\right]-\frac{\partial}{\partial x}[\widetilde{b}(x, k, t) u(x, k, t)], \quad k \in \mathcal{M} .
$$


In what follows, we will prove the smoothness of $\varphi_{i}$ for $0 \leq i \leq n+2$ and the exponential decay of $\psi_{i}$ for $0 \leq i \leq n+1$, which implies the desired error bound by Lemma4.13. Let us consider the layer terms by starting with some lemmas.

LEMma 4.1. For each $k \in \mathcal{M}$, there exists a unique solution $\mu_{k}$ to the following equations:

$$
\widetilde{\mathcal{L}}_{k}^{*}(x, t) \mu_{k}(x, t)=0, \quad \int_{0}^{1} \mu_{k}(x, t) d x=1, \quad \mu_{k}(0, t)=\mu_{k}(1, t) .
$$

REMARK 4.2. The $\mu_{k}(\cdot, \cdot)$ above is said to be a quasi-stationary density. By the smoothness and the periodicity of the boundary conditions in (4.43), the function $\mu_{k}$ defined above also satisfies $\frac{\partial}{\partial x}\left(\widetilde{a}(0, k, t) \mu_{k}(0, t)\right)=\frac{\partial}{\partial x}\left(\widetilde{a}(1, k, t) \mu_{k}(1, t)\right)$.

Definition 4.3. For any functions $\xi(x), \zeta(x)$ on $[0,1]$, define

$$
[\xi, \zeta]=\left(\int_{0}^{1} \xi_{1}(x) \zeta_{1}(x) d x, \ldots, \int_{0}^{1} \xi_{m}(x) \zeta_{m}(x) d x\right)^{\prime} \text { and }\langle\xi, \zeta\rangle=\sum_{k=1}^{m} \int_{0}^{1} \xi_{k}(x) \zeta_{k}(x) d x
$$

LEMmA 4.4. Let $X_{x}^{s}(k, \tau)$ be a Markov process corresponding to the generator $\widetilde{\mathcal{L}}_{k}(x, s)$ and $\omega_{k}(x)$ be a bounded measurable real-valued function. Then

$$
\left|E \omega_{k}\left(X_{x}^{s}(k, \tau)\right)-\int_{0}^{1} \omega_{k}(x) \mu_{k}(x, s) d x\right| \leq C \mathrm{e}^{-\gamma \tau}
$$

Proof. The quasi-stationary density function $\mu_{k}$ of the diffusion process verifies the so-called Doeblin condition that implies the desired result.

Lemma 4.5. For each $k \in \mathcal{M}$, consider the Poisson equation with periodic boundary conditions

$$
\widetilde{\mathcal{L}}_{k}(x, t) \phi(x, k, t)=\zeta_{k}(x, t), \quad \phi(0, k, t)=\phi(1, k, t), \quad \frac{\partial}{\partial x} \phi(0, k, t)=\frac{\partial}{\partial x} \phi(1, k, t) .
$$

Then the only solution to $\widetilde{\mathcal{L}}_{k} \phi(x, k, t)=0$ is $\phi(x, k, t)=\varphi_{k}(t)$. Moreover, (4.44) has a solution if and only if $[\zeta(\cdot, t), \mu(\cdot, t)]=0$.

Proof. 1. Assume $\phi(0, k, t)=\phi(1, k, t)=\varphi_{k}(t)$. Put $\xi_{k}(x, t)=\phi(x, k, t)-\varphi_{k}(t)$. Then

$$
\widetilde{\mathcal{L}}(x, k, t) \xi_{k}(x, t)=0, \quad \xi_{k}(0, t)=\xi_{k}(1, t)=0
$$

It could be verified by the maximum principle for the elliptic operator $\widetilde{\mathcal{L}}$ that $\xi_{k}=0$; see [3. Chapter 6]. So $\phi(x, k, t)=\varphi_{k}(t)$. That is, $\phi(x, k, t)$ is independent of $x$.

2. If (4.44) is solvable, $\left\langle\zeta_{k}(\cdot, t), \mu_{k}(\cdot, t)\right\rangle=\left\langle\widetilde{\mathcal{L}}_{k}(\cdot, t) \phi_{k}(\cdot, t), \mu_{k}(\cdot, t)\right\rangle=\left\langle\phi_{k}(\cdot, t), \widetilde{\mathcal{L}}_{k}^{*}(\cdot, t)\right.$ $\left.\mu_{k}(\cdot, t)\right\rangle=\left\langle\phi_{k}(\cdot, t), 0\right\rangle=0$. Thus $[\zeta(\cdot, t), \mu(\cdot, t)]=0$. Conversely, assume $[\zeta(\cdot, t), \mu(\cdot, t)]=$ 0 . Under the uniform-ellipticity condition, it can be shown that the following equation

$$
\widetilde{\mathcal{L}}_{k}(x, t) \phi(x, k, t)=\zeta_{k}(x, t), \phi(0, k, t)=\phi(1, k, t)
$$


has a solution. In view of (4.2) and using integration by parts,

$$
\begin{aligned}
& \left\langle\zeta_{k}(\cdot, t), \mu_{k}(\cdot, t)\right\rangle=\int_{0}^{1}\left(\widetilde{\mathcal{L}}_{k}(x, t) \phi_{k}(x, t)\right) \mu_{k}(x, t) d x \\
& =\widetilde{a}(1, k, t) \mu_{k}(1, t) \frac{\partial}{\partial x} \phi_{k}(1, t)-\widetilde{a}(0, k, t) \mu_{k}(0, t) \frac{\partial}{\partial x} \phi_{k}(0, t)+\widetilde{b}(1, k, t) \mu_{k}(1, t) \phi_{k}(1, t) \\
& -\widetilde{b}(0, k, t) \mu_{k}(0, t) \phi_{k}(0, t)-\phi_{k}(1, t) \frac{\partial}{\partial x}\left(\widetilde{a}(1, k, t) \mu_{k}(1, t)\right)+\phi_{k}(0, t) \frac{\partial}{\partial x}\left(\widetilde{a}(0, k, t) \mu_{k}(0, t)\right) \\
& +\int_{0}^{1} \phi_{k}(x, t)\left(\widetilde{\mathcal{L}}_{k}^{*}(x, t) \mu_{k}(x, t)\right) d x=\widetilde{a}(0, k, t) \mu_{k}(0, t)\left(\frac{\partial}{\partial x} \phi_{k}(1, t)-\frac{\partial}{\partial x} \phi_{k}(0, t)\right) .
\end{aligned}
$$

Since $\left\langle\zeta_{k}(\cdot, t), \mu_{k}(\cdot, t)\right\rangle=0$ and $\widetilde{a}(0, k, t) \mu_{k}(0, t)>0$, we obtain $\frac{\partial}{\partial x} \phi_{k}(1, t)=\frac{\partial}{\partial x} \phi_{k}(0, t)$. The proof is concluded.

4.1. Leading term $\phi_{0}(x, t)$ and zero-order terminal layer term $\psi_{0}(x, \tau)$. Note that (4.41) gives $\widetilde{\mathcal{L}}(x, t) \phi_{0}(x, t)=0$, which, by Lemma 4.5, implies

$$
\phi_{0}(x, t)=\varphi_{0}(t) .
$$

Moreover, we derived from (4.41) that $\widetilde{\mathcal{L}}(x, t) \phi_{1}(x, t)=-\frac{\partial \phi_{0}(x, t)}{\partial t}-(\widehat{\mathcal{L}}+Q)(x, t) \phi_{0}(x, t)$ $=-\dot{\varphi}_{0}(t)-Q(x, t) \varphi_{0}(t)$. Again, Lemma 4.5 implies that

$$
\dot{\varphi}_{0}(t)+\left[Q(x, t) \varphi_{0}(t), \mu(x, t)\right]=\left[\dot{\varphi}_{0}(t), \mu(x, t)\right]+\left[Q(x, t) \varphi_{0}(t), \mu(x, t)\right]=0 .
$$

Then $\varphi_{0}(T)$ is to be determined. Also, the zero-order terminal layer term is uniquely determined by

$$
\frac{\partial \psi_{0}(x, \tau)}{\partial \tau}=\widetilde{\mathcal{L}}(x, T) \psi_{0}(x, \tau), \quad \psi_{0}(x, 0)=g(x)-\phi_{0}(x, T) .
$$

Then $\psi_{0}(x, \tau)=E \psi_{0}\left(X_{x}^{T}(\tau), 0\right)$, where $X_{x}^{T}(\tau)$ is a Markov process corresponding to the generator $\widetilde{\mathcal{L}}(x, T)$. We demand $\lim _{\tau \rightarrow \infty} \psi_{0}(x, \tau)=0$. By Lemma 4.4, we obtain

$$
\left[\psi_{0}(\cdot, 0), \mu(\cdot, T)\right]=0
$$

which is equivalent to

$$
\varphi_{0}(T)=[g(\cdot), \mu(\cdot, T)] .
$$

Hence $\phi_{0}(x, t)$ is uniquely determined by (4.45), (4.46), and (4.49).

4.2. Higher-order terms. Before proceeding further, we need to verify the following lemmas.

Lemma 4.6. Let $\psi(x, \tau)$ be a solution of

$$
\frac{\partial \psi(x, \tau)}{\partial \tau}=\widetilde{\mathcal{L}}(x, t) \psi(x, \tau)+r(x, \tau), \quad \psi(x, 0)=\omega(x)
$$

where $\omega(x)$ is a l-dimensional vector function and $r(x, \tau)$ decays exponentially fast; i.e., there exist $C, \gamma>0$ such that $\sup _{x \in[0,1]}|r(x, \tau)| \leq C \mathrm{e}^{-\gamma \tau}$. Then

$$
\left|\psi(x, \tau)-[\omega(\cdot), \mu(\cdot, t)]-\int_{0}^{\infty}[r(\cdot, s), \mu(\cdot, t)] d s\right| \leq C \mathrm{e}^{-\gamma \tau}
$$


Proof. In fact, for each $k \in \mathcal{M}, \psi(x, k, \tau)$ satisfies

$$
\frac{\partial \psi(x, k, \tau)}{\partial \tau}=\widetilde{\mathcal{L}}_{k}(x, t) \psi(x, k, \tau)+r_{k}(x, \tau), \quad \psi(x, 0)=\omega_{k}(x) .
$$

Thus $\psi(x, k, \tau)=E \omega_{k}\left(X_{x}(k, \tau)\right)+\int_{0}^{\tau} \operatorname{Er}_{k}\left(X_{x}(k, \tau-s), s\right) d s$, where $X_{x}(k, \tau)$ is the diffusion process associated with the generator $\widetilde{\mathcal{L}}_{k}(x, t)$ satisfying $X_{x}(k, 0)=x$. It follows from Lemma 4.4 that $\left|E \omega_{k}\left(X_{x}(k, \tau)\right)-\left\langle\omega_{k}(\cdot), \mu_{k}(\cdot, t)\right\rangle\right| \leq C \mathrm{e}^{-\gamma \tau}$. Moreover,

$$
\begin{gathered}
\left|\int_{0}^{\tau} \operatorname{Er}_{k}\left(X_{x}(k, \tau-s), s\right) d s-\int_{0}^{\infty}\left\langle r_{k}(\cdot, s), \mu_{k}(\cdot, t)\right\rangle d s\right| \\
\leq\left|\int_{0}^{\tau / 2} \operatorname{Er}_{k}\left(X_{x}(k, \tau-s), s\right) d s-\int_{0}^{\tau / 2}\left\langle r_{k}(\cdot, s), \mu_{k}(\cdot, t)\right\rangle d s\right| \\
\quad+\int_{\tau / 2}^{\tau}\left|E r_{k}\left(X_{x}(k, \tau-s), s\right)\right| d s+\int_{\tau / 2}^{\infty}\left|\left\langle r_{k}(\cdot, s), \mu_{k}(\cdot, t)\right\rangle\right| d s \\
\leq C \mathrm{e}^{-\gamma \tau}+\int_{\tau / 2}^{\tau} C \mathrm{e}^{-\gamma(\tau-s)} d s+\int_{\tau / 2}^{\infty} C \mathrm{e}^{-\gamma s} d s \leq C \mathrm{e}^{-\gamma \tau} .
\end{gathered}
$$

The proof of the lemma is completed.

By using (4.41), we have

$$
\widetilde{\mathcal{L}}(x, t) \phi_{i}(x, t)=\varsigma_{i-1}(x, t) .
$$

By Lemma 4.5, we arrive at

$$
\phi_{i}(x, t)=\varphi_{i}(t)+\widehat{\varphi}_{i}(x, t),
$$

where $\widehat{\varphi}_{i}(x, t)$ is a particular solution of (4.50) satisfying

$$
\left[\widehat{\varphi}_{i}(\cdot, t), \mu(\cdot, t)\right]=0 .
$$

Denote $\bar{a}(x, k, t)=2 \widetilde{a}^{-1}(x, k, t) \widetilde{b}(x, k, t)$ and $\bar{\varsigma}_{i-1}(x, k, t)=\widetilde{a}^{-1}(x, k, t) \varsigma_{i-1}(x, k, t)$. Then

$$
\frac{\partial^{2} \widehat{\varphi}_{i}(x, k, t)}{\partial x^{2}}+\bar{a}(x, k, t) \frac{\partial \widehat{\varphi}_{i}(x, k, t)}{\partial x}=\bar{\varsigma}_{i-1}(x, k, t), \quad \widehat{\varphi}_{i}(0, k, t)=\widehat{\varphi}_{i}(1, k, t) .
$$

Then we obtain

$$
\widehat{\varphi}_{i}(x, k, t)=\widetilde{\varphi}_{i}(k, t)+\bar{\varphi}_{i}(k, t) \int_{0}^{x} \mathrm{e}^{-\rho_{k}(y, t)} d y+\int_{0}^{x} \int_{0}^{y} \mathrm{e}^{\rho_{k}(z, t)-\rho_{k}(y, t)} \bar{\varsigma}_{i-1}(z, k, t) d z d y,
$$

where $\rho_{k}(x, t)=\int_{0}^{x} \bar{a}(y, k, t) d y$. By the periodicity of $\widehat{\varphi}_{i}(\cdot, t)$ (i.e. $\left.\widehat{\varphi}_{i}(0, t)=\widehat{\varphi}_{i}(1, t)\right)$, we deduce

$$
\bar{\varphi}_{i}(t)=-\frac{\int_{0}^{1} \int_{0}^{y} \mathrm{e}^{\rho_{k}(z, t)-\rho_{k}(y, t)} \varsigma_{i-1}(z, t) d z d y}{\int_{0}^{1} \mathrm{e}^{-\rho_{k}(y, t)} d y} .
$$

Moreover, it follows from (4.52) that

$$
\begin{aligned}
\widetilde{\varphi}_{i}(k, t)= & -\int_{0}^{1} \int_{0}^{x} \bar{\varphi}_{i}(k, t) \mathrm{e}^{-\rho_{k}(y, t)} \mu(x, k, t) d y d x \\
& -\int_{0}^{1} \int_{0}^{x} \int_{0}^{y} \mathrm{e}^{\rho_{k}(z, t)-\rho_{k}(y, t)} \bar{\varsigma}_{i-1}(z, k, t) \mu(x, k, t) d z d y d x .
\end{aligned}
$$


On the other hand,

$$
\begin{aligned}
\widetilde{\mathcal{L}}(x, t) \phi_{i+1}(x, t) & =-\frac{\partial}{\partial t} \phi_{i}(x, t)-(\widehat{\mathcal{L}}+Q)(x, t) \phi_{i}(x, t) \\
& =-\dot{\varphi}_{i}(t)-\frac{\partial \widehat{\varphi}_{i}(x, t)}{\partial t}-Q(x, t) \varphi_{i}(t)-(\widehat{\mathcal{L}}+Q)(x, t) \widehat{\varphi}_{i}(x, t) .
\end{aligned}
$$

Using Lemma 4.5, we obtain

$$
\dot{\varphi}_{i}(t)+\left[Q(x, t) \varphi_{i}(t), \mu(x, t)\right]=\left[-\frac{\partial \widehat{\varphi}_{i}(x, t)}{\partial t}-(\widehat{\mathcal{L}}+Q)(x, t) \widehat{\varphi}_{i}(x, t), \mu(x, t)\right] .
$$

The terminal layer term is uniquely determined by

$$
\frac{\partial \psi_{i}(x, \tau)}{\partial \tau}=\widetilde{\mathcal{L}}(x, T) \psi_{i}(x, \tau)+r_{i}(x, \tau), \quad \psi_{i}(x, 0)=-\phi_{i}(x, T) .
$$

Denote by $X_{x}^{T}(k, \tau)$ the Markov process corresponding to the generator $\widetilde{\mathcal{L}}_{k}(x, T)$. Then

$$
\psi_{i}(x, k, \tau)=E \psi_{i}\left(X_{x}^{T}(k, \tau), k, 0\right)+\int_{0}^{\tau} E r_{i}\left(X_{x}^{T}(k, \tau-s), k, s\right) d s .
$$

Furthermore, by demanding $\lim _{\tau \rightarrow \infty} \psi_{i}(x, \tau)=0$ and using Lemma 4.4, we obtain

$$
\left[\psi_{i}(\cdot, 0), \mu(\cdot, T)\right]+\int_{0}^{\infty}\left[r_{i}(\cdot, s), \mu(\cdot, T)\right] d s=0 .
$$

By virtue of the initial condition in (4.57), we arrive at

$$
\varphi_{i}(T)=\int_{0}^{\infty}\left[r_{i}(\cdot, s), \mu(\cdot, T)\right] d s .
$$

Thus $\phi_{i}(x, t)$ is uniquely determined by (4.51), (4.53), (4.56) and (4.59).

Lemma 4.7. There exists a Green function for the following problem:

$$
\frac{\partial \psi}{\partial t}=\widetilde{\mathcal{L}}(x, t) \psi, \quad \psi(0, t)=\psi(1, t), \quad \psi(x, 0)=\omega(x) .
$$

Proof. Let $\widehat{G}$ be the Green function for the corresponding parabolic equation in the unbounded domain (the whole space $\mathbb{R}$ ). Then there exist positive constants $C_{1}, C_{2}, K_{1}$, and $K_{2}$, such that for all $x, y \in \mathbb{R}$ and $t>s$,

$$
C_{1} F_{1}(t-s, y-x) \leq \widehat{G}(s, x, t, y) \leq C_{2} F_{2}(t-s, y-x) .
$$

Here for $\widetilde{h}=1,2, F_{\widetilde{h}}(y, t)$ is the fundamental solution of the equation $K_{\widetilde{h}} \Delta w=\frac{\partial w}{\partial t}$, where $\Delta w$ denotes the Laplacian of $w$. Define $G(s, x, t, y)=\sum_{\iota=-\infty}^{\infty} \widehat{G}(s, x, t, y+\iota)$. In view of (4.60), $G$ is well-defined, since when $t>s$,

$$
G(s, x, t, y) \leq C_{2} \sum_{\iota=-\infty}^{\infty} \frac{1}{\sqrt{4 \pi K_{2}(t-s)}} \exp \left(-\frac{|y+\iota-x|^{2}}{4 K_{2}(t-s)}\right) \leq \infty .
$$

Moreover, by virtue of estimates on the derivatives of $\widehat{G}$, there exist $C>0$ and $K>0$ such that

$$
\begin{aligned}
\left|\frac{\partial^{h}}{\partial y^{h}} \widehat{G}(s, x, t, y)\right| & \leq C \frac{1}{(t-s)^{(1+h) / 2}} \exp \left(-\frac{K|y-x|^{2}}{(t-s)}\right), \\
\left|\frac{\partial}{\partial t} \widehat{G}(s, x, t, y)\right| & \leq C \frac{1}{(t-s)^{3 / 2}} \exp \left(-\frac{K|y-x|^{2}}{(t-s)}\right) .
\end{aligned}
$$


Thus $G$ is differentiable with respect to $y$ and $t$, and the series

$$
\frac{\partial G}{\partial t}=\sum_{\iota=-\infty}^{\infty} \frac{\partial \widehat{G}(s, x, t, y+\iota)}{\partial t} \text { and } \frac{\partial^{h} G}{\partial t^{h}}=\sum_{\iota=-\infty}^{\infty} \frac{\partial^{h} \widehat{G}(s, x, t, y+\iota)}{\partial t^{h}}
$$

converge uniformly. Furthermore, $G$ is periodic with period 1 and satisfies the differential equation.

REMARK 4.8. If $\widetilde{\mathcal{L}}(x, t)$ does not depend on $t$, then we can write the Green function as $G(x, t-s, y)$. In this case, the solution has the form $\psi(x, t)=\int_{0}^{1} G(x, t, y) \omega(y) d y$.

Lemma 4.9. Consider

$$
\frac{\partial \psi}{\partial t}=\widetilde{\mathcal{L}}(x, T) \psi, \quad \psi(0, t)=\psi(1, t), \quad \psi(x, 0)=\omega(x) .
$$

Then there exists an invariant density $\mu(x)$ such that for some $\gamma>0$ and for $h=0,1,2$,

$$
\sup _{x \in[0,1]}\left|\frac{\partial^{h} G(y, \tau, x)}{\partial x^{h}}-\frac{\partial^{h} \mu(x)}{\partial x^{h}}\right| \leq C \mathrm{e}^{-\gamma \tau}
$$

Proof. We have $\sup _{x \in[0,1]}|G(x, \tau, y)-\mu(y)| \leq C \mathrm{e}^{-\gamma \tau}$ (see Lemma 5.1, [9]). Then

$$
\begin{aligned}
& \left|\frac{\partial^{h}}{\partial y^{h}}(G(x, \tau, y)-\mu(y))\right|=\left|\frac{\partial^{h}}{\partial y^{h}}\left(\int_{0}^{1} G(x, \tau-1, z) G(z, 1, y)-\mu(y)\right)\right| \\
& \quad=\left|\frac{\partial^{h}}{\partial y^{h}} \int_{0}^{1}(G(x, \tau-1, z)-\mu(z)) G(z, 1, y)\right| \\
& \quad=\left|\int_{0}^{1}(G(x, \tau-1, z)-\mu(z)) \frac{\partial^{h} G(z, 1, y)}{\partial y^{h}}\right| \\
& \quad \leq C \mathrm{e}^{-\gamma \tau} .
\end{aligned}
$$

Since $C$ does not depend on $x$, we obtain the desired result.

Lemma 4.10. For $i=0, \ldots, n+2, \phi_{i} \in C^{2(n+2), n+2-i}([0,1] \times[0, T])$.

Proof. First of all, from (4.43), we obtain $\mu \in C^{2(n+2), n+2}([0,1] \times[0, T])$. We will prove this lemma by induction. For $i=0$, (4.41) implies $\phi_{0} \in C^{2(n+2), n+2}([0,1] \times$ $[0, T])$. Now assume $\phi_{j} \in C^{2(n+2), n+2-j}([0,1] \times[0, T])$ for $j \leq i$. In view of (4.41), $\varsigma_{i} \in C^{2(n+1), n+1-i}([0,1] \times[0, T])$. Then, by (4.53), $\widehat{\varphi}_{i+1} \in C^{2(n+2), n+1-i}([0,1] \times[0, T])$. On the other hand, we can conclude from (4.56) that $\varphi_{i+1} \in C^{n+1-i}([0, T])$. Therefore, $\phi_{i+1} \in C^{2(n+2), n+1-i}([0,1] \times[0, T])$. This completes the proof of this lemma.

Lemma 4.11. Let $0 \leq i \leq n+1$ be a fixed integer. For a nonnegative integer $h$ with $0 \leq$ $h \leq 2(n+2-i)$, put $f_{i}^{h}(x, \tau)=\frac{\partial^{h} \psi_{i}(x, \tau)}{\partial x^{h}}$. Assume for any $\tau, x,\left|\psi_{i}(x, \tau)\right| \leq C \mathrm{e}^{-\gamma \tau}$ and $\max _{h=0, \ldots, 2(n+2-i)}\left|\frac{\partial^{h} r_{i}(x, \tau)}{\partial x^{h}}\right| \leq C \mathrm{e}^{-\gamma \tau}$. Then for any $\tau, x, \max _{h=0, \ldots, 2(n+2-i)}\left|f_{i}^{h}(x, \tau)\right|$ $\leq C \mathrm{e}^{-\gamma \tau}$. 
Proof. First, $\left|f_{i}^{0}(x, \tau)\right|=\left|\psi_{i}(x, \tau)\right| \leq C \mathrm{e}^{-\gamma \tau}$. Suppose for any $\widetilde{h}<h,\left|f_{i}^{\widetilde{h}}(x, \tau)\right| \leq$ $C \mathrm{e}^{-\gamma \tau}$. Then (4.42) implies that

$$
\begin{aligned}
\frac{\partial f_{i}^{h}(x, \tau)}{\partial \tau}= & \widetilde{\mathcal{L}}(x, T) f_{i}^{h}(x, \tau)+\frac{\partial^{h} r_{i}(x, \tau)}{\partial x^{h}} \\
& +\sum_{\widetilde{h}=0}^{h-1}\left(\begin{array}{l}
\frac{h}{h} \\
h
\end{array}\right)\left(\frac{\partial^{h-\widetilde{h}} \widetilde{a}(x, T)}{\partial x^{h-\widetilde{h}}} f_{i}^{\widetilde{h}+2}(x, \tau)+\frac{\partial^{h-\widetilde{h}} \widetilde{b}(x, T)}{\partial x^{h-\widetilde{h}}} f_{i}^{\widetilde{h}+1}(x, \tau)\right) \\
\stackrel{\text { def }}{=} & \widetilde{\mathcal{L}}(x, T) f_{i}^{h}(x, \tau)+\frac{\partial^{h} r_{i}(x, \tau)}{\partial x^{h}}+\widetilde{f}_{i}^{h}(x, \tau), \\
f_{i}^{h}(x, 0)= & \frac{\partial^{h} \psi(x, 0)}{\partial x^{h}} .
\end{aligned}
$$

We claim that $\widehat{f}_{i}^{h} \stackrel{\text { def }}{=} \int_{0}^{1} f_{i}^{h}(x, 0) \mu(x, T) d x+\int_{0}^{\infty} \int_{0}^{1} \widetilde{f}_{i}^{h}(x, s) \mu(x, T) d x d s=0$. Let $G_{i}^{h}$ and $\mu_{i}^{h}$ be the Green function for equation (4.61) and its associated invariant density respectively. Put $w_{i}^{h}(x, s)=G_{i}^{h}(x, s)-\mu_{i}^{h}(x, T)$. Then

$$
\begin{aligned}
& f_{i}^{h}(x, \tau)=\int_{0}^{1} G_{i}^{h}(x, \tau, y) f_{i}^{h}(y, 0) d y+\int_{0}^{\tau} \int_{0}^{1} G_{i}^{h}(x, \tau-s, y) \frac{\partial^{h} r_{i}(y, s)}{\partial x^{h}} d x d s \\
& +\int_{0}^{\tau} \int_{0}^{1} \sum_{\widetilde{h}=0}^{h-1}\left(\begin{array}{l}
h \\
\widetilde{h}
\end{array}\right) G_{i}^{h}(x, \tau-s, y)\left(\frac{\partial^{h-\widetilde{h}} \widetilde{a}(y, T)}{\partial y^{h-\widetilde{h}}} f_{i}^{\widetilde{h}+2}(y, s)+\frac{\partial^{h-\widetilde{h}} \widetilde{b}(y, T)}{\partial y^{h-\widetilde{h}}} f_{i}^{\widetilde{h}+1}(y, s)\right) d y d s \\
& =\int_{0}^{1} w_{i}^{h}(x, \tau, y) f_{i}^{h}(y, 0) d y+\int_{0}^{\tau} \int_{0}^{1} w_{i}^{h}(x, \tau-s, y) \frac{\partial^{h} r_{i}(y, s)}{\partial x^{h}} d y d s \\
& +\int_{0}^{\tau} \int_{0}^{1} \sum_{\widetilde{h}=0}^{h-1}\left(\begin{array}{l}
h \\
\widetilde{h}
\end{array}\right) w_{i}^{h}(x, \tau-s, y) \frac{\partial^{h-\widetilde{h}} \widetilde{a}(y, T)}{\partial y^{h-\widetilde{h}}} f_{i}^{\widetilde{h}+2}(y, s) d y d s \\
& +\int_{0}^{\tau} \int_{0}^{1} \sum_{\widetilde{h}=0}^{h-1}\left(\begin{array}{l}
h \\
\widetilde{h}
\end{array}\right) w_{i}^{h}(x, \tau-s, y) \frac{\partial^{h-\widetilde{h}} \widetilde{b}(y, T)}{\partial y^{h-\widetilde{h}}} f_{i}^{\widetilde{h}+1}(y, s) d y d s \\
& +\int_{\tau}^{\infty} \int_{0}^{1} \mu_{i}^{h}(y, T) \frac{\partial^{h} r_{i}(y, s)}{\partial x^{h}} d y d s+\int_{\tau}^{\infty} \int_{0}^{1} \sum_{\widetilde{h}=0}^{h-1}\left(\begin{array}{l}
h \\
\widetilde{h}
\end{array}\right) \mu_{i}^{h}(y, T) \frac{\partial^{h-\widetilde{h}} \widetilde{a}(y, T)}{\partial y^{h-\widetilde{h}}} f_{i}^{\widetilde{h}+2}(y, s) d y d s \\
& +\int_{\tau}^{\infty} \int_{0}^{1} \sum_{\widetilde{h}=0}^{h-1}\left(\begin{array}{l}
h \\
\widetilde{h}
\end{array}\right) \mu_{i}^{h}(y, T) \frac{\partial^{h-\widetilde{h}} \widetilde{b}(y, T)}{\partial y^{h-\widetilde{h}}} f_{i}^{\widetilde{h}+1}(y, s) d y d s .
\end{aligned}
$$

On the other hand, by Lemma 4.9, for all $s>0, \max _{j=0,1,2} \sup _{x \in[0,1]}\left|\frac{\partial^{j} w_{i}^{h}(x, s)}{\partial y^{j}}\right| \leq C \mathrm{e}^{-\gamma s}$. Therefore, 


$$
\begin{aligned}
& \left|\int_{0}^{\tau} \int_{0}^{1} \sum_{\widetilde{h}=0}^{h-1}\left(\begin{array}{l}
h \\
h
\end{array}\right) w_{i}^{h}(x, \tau-s, y) \frac{\partial^{h-\widetilde{h}} \widetilde{a}(y, T)}{\partial y^{h-\widetilde{h}}} f_{i}^{\widetilde{h}+2}(y, s) d y d s\right| \\
& =\left|\int_{0}^{\tau} \int_{0}^{1} \sum_{\widetilde{h}=0}^{h-1}\left(\begin{array}{l}
h \\
\widetilde{h}
\end{array}\right) \frac{\partial^{2}}{\partial y^{2}}\left(w_{i}^{h}(x, \tau-s, y) \frac{\partial^{h-\widetilde{h}} \widetilde{a}(y, T)}{\partial y^{h-\widetilde{h}}}\right) f_{i}^{\widetilde{h}}(y, s) d y d s\right| \\
& \leq \int_{0}^{\tau} C \mathrm{e}^{-\gamma(\tau-s)} \mathrm{e}^{-\gamma s} d s \leq C \mathrm{e}^{-\widetilde{\gamma} \tau},
\end{aligned}
$$

and also the following inequality

$$
\begin{aligned}
& \left|\int_{\tau}^{\infty} \int_{0}^{1} \sum_{\widetilde{h}=0}^{h-1}\left(\begin{array}{l}
h \\
\widetilde{h}
\end{array}\right) \mu_{i}^{h}(y, T) \frac{\partial^{h-\widetilde{h}} \widetilde{a}(y, T)}{\partial y^{h-\widetilde{h}}} f_{i}^{\widetilde{h}+2}(y, s) d y d s\right| \\
& =\left|\int_{\tau}^{\infty} \int_{0}^{1} \sum_{\widetilde{h}=0}^{h-1}\left(\begin{array}{l}
h \\
\widetilde{h}
\end{array}\right) \frac{\partial^{2}}{\partial y^{2}}\left(\mu_{i}^{h}(y, T) \frac{\partial^{h-\widetilde{h}} \widetilde{a}(y, T)}{\partial y^{h-\widetilde{h}}}\right) f_{i}^{\widetilde{h}}(y, s) d y d s\right| \\
& \leq \int_{\tau}^{\infty} C \mathrm{e}^{-\gamma s} d s=C \mathrm{e}^{-\gamma \tau}
\end{aligned}
$$

for some $0<\widetilde{\gamma}<\gamma$. Similar results could be obtained for the other terms. Then $\sup _{x \in[0,1]}\left|f_{i}^{h}(x, \tau)\right| \leq C \mathrm{e}^{-\bar{\gamma} \tau}$ for some $\bar{\gamma}>0$. Thus the desired result follows immediately by induction. Now we will verify the above claim. In fact, for any $\widetilde{h} \leq h$,

$$
\begin{aligned}
\frac{\partial f_{i}^{\widetilde{h}-1}(x, s)}{\partial s} & =\frac{\partial^{\widetilde{h}-1}}{\partial x^{\widetilde{h}-1}}\left(\widetilde{\mathcal{L}}(x, T) \psi_{i}(x, s)\right)+\frac{\partial^{\widetilde{h}-1} r_{i}(x, s)}{\partial x^{\widetilde{h}-1}} \\
& =\sum_{\iota=0}^{\widetilde{h}-1}\left(\begin{array}{c}
\widetilde{h}-1 \\
\iota
\end{array}\right)\left(\frac{\partial^{\widetilde{h}-1-\iota}}{\partial x^{\widetilde{h}-1-\iota}} \widetilde{\mathcal{L}}(x, T)\right) f_{i}^{\iota}(x, s)+\frac{\partial^{\widetilde{h}-1} r_{i}(x, s)}{\partial x^{\widetilde{h}-1}}
\end{aligned}
$$

Integrating the above equation, we have

$$
-f_{i}^{h-1}(x, 0)=\int_{0}^{\infty}\left(\sum_{\widetilde{h}=0}^{h-1}\left(\begin{array}{c}
h-1 \\
\widetilde{h}
\end{array}\right)\left(\frac{\partial^{h-1-\widetilde{h}}}{\partial x^{h-1-\widetilde{h}}} \widetilde{\mathcal{L}}(x, T)\right) f_{i}^{\widetilde{h}}(x, s)+\frac{\partial^{h-1} r_{i}(x, s)}{\partial x^{h-1}}\right) d s
$$


Differentiating with respect to $x$ and noting that $\left(\begin{array}{c}h-1 \\ \widetilde{h}\end{array}\right)+\left(\begin{array}{c}h-1 \\ \widetilde{h}-1\end{array}\right)=\left(\begin{array}{l}h \\ \breve{h}\end{array}\right)$, we obtain

$$
\begin{aligned}
& -f_{i}^{h}(x, 0)=\int_{0}^{\infty}\left(\sum _ { \widetilde { h } = 0 } ^ { h - 1 } ( \begin{array} { c } 
{ h - 1 } \\
{ \widetilde { h } }
\end{array} ) \left(\left(\frac{\partial^{h-\widetilde{h}}}{\partial x^{h-\widetilde{h}}} \widetilde{\mathcal{L}}(x, T)\right) f_{i}^{\widetilde{h}}(x, s)\right.\right.
\end{aligned}
$$

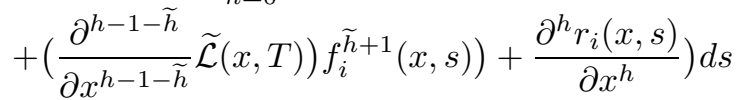

$$
\begin{aligned}
& =\int_{0}^{\infty}\left(\sum_{\widetilde{h}=0}^{h-1}\left(\begin{array}{c}
h-1 \\
\widetilde{h}
\end{array}\right)\left(\frac{\partial^{h-\widetilde{h}}}{\partial x^{h-\widetilde{h}}} \widetilde{\mathcal{L}}(x, T)\right) f_{i}^{\widetilde{h}}(x, s)\right. \\
& \left.+\sum_{\widetilde{h}=1}^{h}\left(\begin{array}{c}
h-1 \\
\widetilde{h}-1
\end{array}\right)\left(\frac{\partial^{h-\widetilde{h}}}{\partial x^{h-\widetilde{h}}} \widetilde{\mathcal{L}}(x, T)\right) f_{i}^{\widetilde{h}}(x, s)+\frac{\partial^{h} r_{i}(x, s)}{\partial x^{h}}\right) d s \\
& =\int_{0}^{\infty}\left(\sum_{\widetilde{h}=0}^{h}\left(\frac{h}{h}\right)\left(\frac{\partial^{h-\widetilde{h}}}{\partial x^{h-\widetilde{h}}} \widetilde{\mathcal{L}}(x, T)\right) f_{i}^{\widetilde{h}}(x, s)+\frac{\partial^{h} r_{i}(x, s)}{\partial x^{h}}\right) d s \\
& =\int_{0}^{\infty}\left(\widetilde{\mathcal{L}}(x, T) f_{i}^{h}(x, s)+\widetilde{f}_{i}^{h}(x, s)\right) d s .
\end{aligned}
$$

Therefore,

$$
-\left\langle f_{i}^{h}(x, 0), \mu(x, T)\right\rangle=\int_{0}^{\infty}\left\langle\widetilde{\mathcal{L}}(x, T) f_{i}^{h}(x, s), \mu(x, T)\right\rangle d s+\int_{0}^{\infty}\left\langle\widetilde{f}_{i}^{h}(x, s), \mu(x, T)\right\rangle d s .
$$

Hence, the claim is proved.

Lemma 4.12. There exist constants $C$ and $0<\widetilde{\gamma}<\gamma$ such that for any $0 \leq i \leq n+1$ and $\tau>0$,

$$
\max _{h=0, \ldots, 2(n+2-i)} \max _{x \in[0,1]}\left|\frac{\partial^{h} \psi_{i}(x, \tau)}{\partial x^{h}}\right| \leq C \mathrm{e}^{-\widetilde{\gamma} \tau} .
$$

As a result, for any $0 \leq i \leq n+1$ and $\tau>0$,

$$
\max _{0 \leq \widetilde{h} \leq n+2}\left|\widetilde{\mathcal{L}}^{(\widetilde{h})}(x, T) \psi_{i}(x, \tau)\right| \leq C \mathrm{e}^{-\widetilde{\gamma} \tau} \text { and } \max _{0 \leq \widetilde{h} \leq n+2}\left|(\widehat{\mathcal{L}}+Q)^{(\widetilde{h})}(x, T) \psi_{i}(x, \tau)\right| \leq C \mathrm{e}^{-\widetilde{\gamma} \tau} .
$$

Proof. First, under condition (4.48) and by Lemma4.6, we have $\left|\psi_{0}(x, \tau)\right|=\mid \psi_{0}(x, \tau)-$ $\left[\psi_{0}(\cdot, 0), \mu(\cdot, T)\right] \mid \leq C \mathrm{e}^{-\gamma \tau}$. Applying Lemma 4.11 with $r_{0}=0$, we verify that (4.62) holds for $i=0$. Assume that for any $j<i, \max _{h=0, \ldots, 2(n+2-j)} \sup _{x \in[0,1]}\left|\frac{\partial^{h} \psi_{j}(x, \tau)}{\partial x^{h}}\right| \leq C \mathrm{e}^{-\gamma \tau}$. Then for some $0<\widetilde{\gamma}<\gamma, \max _{h=0, \ldots, 2(n+2-i)} \sup _{x \in[0,1]}\left|\frac{\partial^{h} r_{i}(x, \tau)}{\partial x^{h}}\right| \leq C \mathrm{e}^{-\gamma \tau}$. Now, under condition (4.58), we again derive from Lemma 4.6 that

$$
\left|\psi_{i}(x, \tau)\right|=\left|\psi_{i}(x, \tau)-\left[\psi_{i}(\cdot, 0), \mu(\cdot, T)\right]-\int_{0}^{\infty}\left[r_{i}(\cdot, t), \mu(\cdot, T)\right] d t\right| \leq C \mathrm{e}^{-\widetilde{\gamma} \tau} .
$$

Thus Lemma 4.11 yields (4.62). This completes the proof by induction.

4.3. Error estimates. For any function $f$, define $\mathcal{D}^{\varepsilon} f=\frac{\partial f}{\partial t}+Q f+\mathcal{L}^{\varepsilon} f$.

Lemma 4.13. Suppose that $\zeta \in C([0,1] \times[0, T])$ is periodic in $x \in[0,1]$, satisfying $\sup _{(x, t) \in[0,1] \times[0, T]}|\zeta(x, t)| \leq C \varepsilon^{\kappa}$. Let $\xi^{\varepsilon}(x, t)$ be a solution to

$$
\mathcal{D}^{\varepsilon} \xi^{\varepsilon}(x, t)=\zeta(x, t), \quad \xi^{\varepsilon}(x, T)=0, \quad \forall x \in[0,1] .
$$


Then $\sup _{(x, t) \in[0,1] \times[0, T]}\left|\xi^{\varepsilon}(x, t)\right| \leq C \varepsilon^{\kappa}$.

Proof. Using $\tau=(T-t) / \varepsilon$ and putting $F^{\varepsilon}(x, \tau)=\xi^{\varepsilon}(x, \varepsilon \tau)$, (4.63) becomes

$$
\frac{\partial F^{\varepsilon}(x, \tau)}{\partial \tau}=(\widetilde{\mathcal{L}}(x, \varepsilon \tau)+\varepsilon \widehat{\mathcal{L}}(x, \varepsilon \tau)) F^{\varepsilon}(x, \tau)+\varepsilon Q(x, \varepsilon \tau) F^{\varepsilon}(x, \tau)+\varepsilon \zeta(x, \varepsilon \tau) .
$$

Since $\widetilde{\mathcal{L}}(x, \varepsilon \tau)+\varepsilon \widehat{\mathcal{L}}(x, \varepsilon \tau)$ is elliptic, there exists a Green's function $G_{\varepsilon}$ such that $F^{\varepsilon}(x, \tau)=\int_{0}^{\tau} \int_{0}^{1} G_{\varepsilon}(s, y, \tau, x) \varepsilon \zeta(y, \varepsilon s) d y d s+\int_{0}^{\tau} \int_{0}^{1} G_{\varepsilon}(s, y, \tau, x) \varepsilon Q(y, \varepsilon s) F^{\varepsilon}(y, s) d y d s$.

We have

$$
\begin{aligned}
& \int_{0}^{1} G_{\varepsilon}(s, y, \tau, x) d y \\
& \leq \int_{0}^{1} \sum_{\iota=-\infty}^{\infty} \frac{1}{\sqrt{4 \pi c(\tau-s)}} \exp \left(-\frac{(x+\iota-y)^{2}}{4 \pi c(\tau-s)}\right) d y \\
& =\int_{-\infty}^{\infty} \frac{1}{\sqrt{4 \pi c(\tau-s)}} \exp \left(-\frac{(x+y)^{2}}{4 \pi c(\tau-s)}\right) d y \\
& =\int_{-\infty}^{\infty} e^{-z^{2}} d z\left(\text { with } z=\frac{x+y}{\sqrt{4 \pi c(\tau-s)}}\right)=\frac{\pi}{2 \sqrt{2}} .
\end{aligned}
$$

Put $f(\tau)=\sup _{x \in[0,1]}\left|F^{\varepsilon}(x, \tau)\right|$. Then $f(\tau) \leq C \varepsilon^{\kappa+1} \tau+C \varepsilon \int_{0}^{\tau} f(s) d s \leq C \varepsilon^{\kappa}$ $+C \varepsilon \int_{0}^{\tau} f(s) d s$. Using Gronwall's inequality, we obtain $f(\tau) \leq C \varepsilon^{\kappa} \exp \left(C \varepsilon \int_{0}^{\tau} d s\right)$ $\leq C \varepsilon^{\kappa}$.

Theorem 4.14. There exists $C>0$ such that

$$
\sup _{(x, t) \in[0,1] \times[0, T]}\left|u^{\varepsilon}(x, t)-\Phi_{n}^{\varepsilon}(x, t)-\Psi_{n}^{\varepsilon}\left(x, \frac{T-t}{\varepsilon}\right)\right| \leq C \varepsilon^{n+1} .
$$

Proof. Put $e^{\varepsilon, \kappa}(x, t)=u^{\varepsilon}(x, t)-\Phi_{\kappa}^{\varepsilon}(x, t)-\Psi_{\kappa}^{\varepsilon}(x, \tau)$. Then $\mathcal{D}^{\varepsilon} u^{\varepsilon}(x, t)=0$ and $\mathcal{D}^{\varepsilon} e^{\varepsilon, \kappa}(x, t)=-\mathcal{D}^{\varepsilon} \Phi_{\kappa}^{\varepsilon}(x, t)-\mathcal{D}^{\varepsilon} \Psi_{\kappa}^{\varepsilon}(x, \tau)$. Moreover

$$
\begin{aligned}
\mathcal{D}^{\varepsilon} \Phi_{\kappa}^{\varepsilon}(x, t) & =\sum_{i=0}^{\kappa} \varepsilon^{i} \dot{\phi}_{i}(x, t)+\sum_{i=0}^{\kappa} \varepsilon^{i-1} \widetilde{\mathcal{L}}(x, t) \phi_{i}(x, t)+\sum_{i=0}^{\kappa} \varepsilon^{i}(\widehat{\mathcal{L}}+Q)(x, t) \phi_{i}(x, t) \\
& =-\varepsilon^{\kappa} \widetilde{\mathcal{L}}(x, t) \phi_{\kappa+1}(x, t)+\underbrace{\varepsilon^{-1} \widetilde{\mathcal{L}}(x, t) \phi_{0}(x, t)}_{0} .
\end{aligned}
$$

By Lemma 4.10, $\left|\mathcal{D}^{\varepsilon} \Phi_{\kappa}^{\varepsilon}(x, t)\right| \leq C \varepsilon^{\kappa}$. Using $\tau, \varepsilon \frac{\partial}{\partial t} \psi_{i}(x, \tau)=-\frac{d}{d \tau} \psi_{i}(x, \tau)$, which yields

$$
\begin{aligned}
\mathcal{D}^{\varepsilon} & \Psi_{\kappa}^{\varepsilon}(x, \tau)=\sum_{i=0}^{\kappa}-\varepsilon^{i-1} \frac{d}{d \tau} \psi_{i}(x, \tau)+\sum_{i=0}^{\kappa} \varepsilon^{i-1} \widetilde{\mathcal{L}}(x, t) \psi_{i}(x, \tau)+\sum_{i=0}^{\kappa} \varepsilon^{i}(\widehat{\mathcal{L}}+Q)(x, t) \psi_{i}(x, \tau) \\
= & \sum_{i=0}^{\kappa} \varepsilon^{i-1}\left(-\widetilde{\mathcal{L}}(x, T) \psi_{i}(x, \tau)-r_{i}(x, \tau)\right)+\sum_{i=0}^{\kappa} \varepsilon^{i-1} \widetilde{\mathcal{L}}(x, t) \psi_{i}(x, \tau) \\
& +\sum_{i=0}^{\kappa} \varepsilon^{i}(\widehat{\mathcal{L}}+Q)(x, t) \psi_{i}(x, \tau) \\
= & \sum_{i=0}^{\kappa} \varepsilon^{i-1}(-\widetilde{\mathcal{L}}(x, T)+\widetilde{\mathcal{L}}(x, t)) \psi_{i}(x, \tau)-\sum_{i=0}^{\kappa} \varepsilon^{i-1} r_{i}(x, \tau)+\sum_{i=0}^{\kappa} \varepsilon^{i}(\widehat{\mathcal{L}}+Q)(x, t) \psi_{i}(x, \tau) .
\end{aligned}
$$


The second term is equal to

$$
\begin{aligned}
& \sum_{i=0}^{\kappa} \varepsilon^{i-1} r_{i}(x, \tau) \\
= & \sum_{i=1}^{\kappa} \varepsilon^{i-1} \sum_{j=0}^{i-1}\left((-\tau)^{i-j} \frac{\widetilde{\mathcal{L}}^{(i-j)}(x, T)}{(i-j) !}+(-\tau)^{i-j-1} \frac{(\widehat{\mathcal{L}}+Q)^{(i-j-1)}(x, T)}{(i-j-1) !}\right) \psi_{j}(x, \tau) \\
= & \sum_{j=0}^{\kappa-1} \sum_{i=j+1}^{\kappa}\left(\varepsilon^{i-1}(-\tau)^{i-j} \frac{\widetilde{\mathcal{L}}^{(i-j)}(x, T)}{(i-j) !}+\varepsilon^{i-1}(-\tau)^{i-j-1} \frac{(\widehat{\mathcal{L}}+Q)^{(i-j-1)}(x, T)}{(i-j-1) !}\right) \psi_{j}(x, \tau) \\
= & \sum_{j=0}^{\kappa-1} \sum_{i=j+1}^{\kappa}\left(\varepsilon^{j-1}(t-T)^{i-j} \frac{\widetilde{\mathcal{L}}^{(i-j)}(x, T)}{(i-j) !}+\varepsilon^{j}(t-T)^{i-j-1} \frac{(\widehat{\mathcal{L}}+Q)^{(i-j-1)}(x, T)}{(i-j-1) !}\right) \psi_{j}(x, \tau) \\
= & \sum_{j=0}^{\kappa-1} \varepsilon^{j-1}\left(\sum_{i=1}^{\kappa-j}(t-T)^{i} \frac{\widetilde{\mathcal{L}}^{(i)}(x, T)}{i !}\right) \psi_{j}(x, \tau) \\
& +\sum_{j=0}^{\kappa-1} \varepsilon^{j}\left(\sum_{i=0}^{\kappa-j-1}(t-T)^{i} \frac{(\widehat{\mathcal{L}}+Q)^{(i)}(x, T)}{i !}\right) \psi_{j}(x, \tau) .
\end{aligned}
$$

Therefore,

$$
\begin{aligned}
\mathcal{D}^{\varepsilon} \Psi_{\kappa}^{\varepsilon}(x, \tau) & =\varepsilon^{\kappa-1}(-\widetilde{\mathcal{L}}(x, T)+\widetilde{\mathcal{L}}(x, t)) \psi_{\kappa}(x, \tau)+\varepsilon^{\kappa}(\widehat{\mathcal{L}}+Q)(x, t) \psi_{\kappa}(x, \tau) \\
& +\sum_{j=0}^{\kappa-1} \varepsilon^{j-1}\left(\widetilde{\mathcal{L}}(x, t)-\sum_{i=0}^{\kappa-j}(t-T)^{i} \frac{\widetilde{\mathcal{L}}^{(i)}(x, T)}{i !}\right) \psi_{j}(x, \tau) \\
& +\sum_{j=0}^{\kappa-1} \varepsilon^{j}\left((\widehat{\mathcal{L}}+Q)(x, t)-\sum_{i=0}^{\kappa-j-1}(t-T)^{i} \frac{(\widehat{\mathcal{L}}+Q)^{(i)}(x, T)}{i !}\right) \psi_{j}(x, \tau) .
\end{aligned}
$$

Using Taylor expansions and Proposition 4.12, we obtain

$$
\begin{aligned}
\left|\mathcal{D}^{\varepsilon} \Psi_{\kappa}^{\varepsilon}(x, \tau)\right| \leq & C \varepsilon^{\kappa-1}|t-T| \mathrm{e}^{-\gamma \tau}+C \varepsilon^{\kappa}+C \sum_{j=0}^{\kappa-1} \varepsilon^{j-1}|t-T|^{\kappa-j+1} \mathrm{e}^{-\gamma \tau} \\
& +C \sum_{j=0}^{\kappa-1} \varepsilon^{j}|t-T|^{\kappa-j} \mathrm{e}^{-\gamma \tau} \\
= & C \varepsilon^{\kappa} \tau \mathrm{e}^{-\gamma \tau}+C \varepsilon^{\kappa}+C \sum_{j=0}^{\kappa-1} \varepsilon^{\kappa} \tau^{\kappa-j+1} \mathrm{e}^{-\gamma \tau}+C \sum_{j=0}^{\kappa-1} \varepsilon^{\kappa} \tau^{\kappa-j} \mathrm{e}^{-\gamma \tau} \leq C \varepsilon^{\kappa}
\end{aligned}
$$

Piecing this together with the estimates on $\mathcal{D}^{\varepsilon} \Phi_{\kappa}(x, t), \sup _{(x, t) \in[0,1] \times[0, T]}\left|\mathcal{D}^{\varepsilon} e^{\varepsilon, \kappa}(x, t)\right| \leq$ $C \varepsilon^{\kappa}$ for $k \leq n+1$. Note the terminal condition $e^{\varepsilon, \kappa}(x, T)=0$. Thus Lemma 4.13 implies $\sup _{(x, t) \in[0,1] \times[0, T]}\left|e^{\varepsilon, \kappa}(x, t)\right| \leq C \varepsilon^{\kappa}$. Taking $\kappa=n+1, \sup _{(x, t) \in[0,1] \times[0, T]}\left|e^{\mathrm{e}, n+1}(x, t)\right|=$ $O\left(\varepsilon^{n+1}\right)$. Finally, note that

$$
e^{\varepsilon, n+1}(x, t)=e^{\varepsilon, n}(x, t)+\varepsilon^{n+1} \phi_{n+1}(x, t)+\varepsilon^{n+1} \psi_{n+1}(x, \tau) .
$$

The continuity of $\phi_{n+1}(x, t)$ and the exponential decay properties of $\psi_{n+1}(x, \tau)$ yield that

$$
\sup _{(x, t) \in[0,1] \times[0, T]}\left|\varepsilon^{n+1} \phi_{n+1}(x, t)+\varepsilon^{n+1} \psi_{n+1}(x, \tau)\right| \leq C \varepsilon^{n+1} .
$$


Substituting this into (4.65), we obtain $\sup _{(x, t) \in[0,1] \times[0, T]}\left|e^{\varepsilon, n}(x, t)\right| \leq C \varepsilon^{n+1}$ as desired.

5. Illustrations and remarks. Asymptotic expansions have been constructed in this paper. In this section, we provide some interpretations of our results.

Consider (2.1) together with (2.2), in which $Q^{\varepsilon}(x)=Q(x) / \varepsilon$ and $Q(x, t)$ is weakly irreducible. Now (2.1) and (2.2) can be written as

$$
\begin{aligned}
& d X^{\varepsilon}(t)=b\left(X^{\varepsilon}(t), \alpha^{\varepsilon}(t)\right) d t+\sigma\left(X^{\varepsilon}(t), \alpha^{\varepsilon}(t)\right) d B(t), \\
& P\left(\alpha^{\varepsilon}(t+\Delta)=\ell \mid \alpha^{\varepsilon}(t)=k, X(t)=x\right)=q_{k \ell}^{\varepsilon}(x) \Delta+o(\Delta) .
\end{aligned}
$$

Using weak convergence methods (see, e.g., [14]), one can show that $X^{\varepsilon}(\cdot)$ converges weakly to $X(\cdot)$ such that $X(\cdot)$ is the solution of

$$
d X(t)=\bar{b}(X(t)) d t+\bar{\sigma}(X(t)) d B(t)
$$

where

$$
\bar{b}(x)=\sum_{i=1}^{m} b(x, i) \nu_{i}(x), \quad \bar{\sigma}(x)=\sqrt{\sum_{i=1}^{m} \sigma^{2}(x, i) \nu_{i}(x)}, \nu(x)=\left(\nu_{1}(x), \ldots, \nu_{m}(x)\right)
$$

is the quasi-stationary distribution. The asymptotic results obtained in this paper give us more than those only obtained by the weak convergence. It provides new insight even for the leading term in the asymptotic expansion.

Suppose that $U(x, \alpha)$ is a smooth functional. Our asymptotic expansions (e.g., Theorem 3.10) and the probabilistic interpretation of the solution of the backward equation enable us to conclude that for any $t>K \varepsilon \ln (1 / \varepsilon)$ and some $K>0$,

$$
E U\left(X^{\varepsilon}(t), \alpha^{\varepsilon}(t)\right) \rightarrow E \bar{U}(X(t))=E \sum_{i=1}^{m} U(X(t), i) \nu_{i}(X(t))
$$

Next, consider $\widetilde{U}(x, \alpha)=1_{A} 1_{B}$ (the indicators of $A$ and $B$, resp.), which can be thought of as an approximation to the smooth function $U$. Then we have $P\left(X^{\varepsilon}(t) \in A, \alpha^{\varepsilon}(t) \in\right.$ $B) \rightarrow \sum_{i \in B} \int_{A} P(X(t) \in d x) \nu_{i}(x)$ as $\varepsilon \rightarrow 0$. In particular, when $A=[0,1], P\left(\alpha^{\varepsilon}(t) \in\right.$ $B) \rightarrow \sum_{i \in B} E \nu_{i}(X(t))$ as $\varepsilon \rightarrow 0$. As a convention, $X^{x, i}(t)$ denotes the process $X(t)$ starting at $X(0)=x$ and $\alpha(t)=i$. By virtue of the Markov property of $\left(X^{\varepsilon}(t), \alpha^{\varepsilon}(t)\right)$, 
for $0<t_{1}<t_{2}$ not depending on $\varepsilon$,

$$
\begin{aligned}
P & \left(\alpha^{\varepsilon, x, i}\left(t_{1}\right) \in A_{1}, \alpha^{\varepsilon, x, i}\left(t_{2}\right) \in A_{2}\right) \\
& =\sum_{i_{1} \in A_{1}} \int_{0}^{1} P\left(X^{\varepsilon, x, i}(t) \in d x_{1}, \alpha^{\varepsilon, x, i}(t)=i_{1}\right) P\left(\alpha^{\varepsilon, x_{1}, i_{1}}\left(t_{2}-t_{1}\right) \in A_{2}\right) \\
& \rightarrow \sum_{i_{1} \in A_{1}} \int_{0}^{1} P\left(X^{x}(t) \in d x_{1}\right) \nu_{i_{1}}\left(x_{1}\right) \sum_{i_{2} \in A_{2}} E \nu_{i_{2}}\left(X^{x_{1}}\left(t_{2}-t_{1}\right)\right) \text { as } \varepsilon \rightarrow 0 \\
& =\sum_{i_{1} \in A_{1}} \sum_{i_{2} \in A_{2}} E \nu_{i_{1}}\left(X^{x}\left(t_{1}\right)\right) E \nu_{i_{2}}\left(X^{X^{x}\left(t_{1}\right)}\left(t_{2}-t_{1}\right)\right) \\
& =\sum_{i_{1} \in A_{1}} \sum_{i_{2} \in A_{2}} E\left[\nu_{i_{1}}\left(X^{x}\left(t_{1}\right)\right) E\left(\nu_{i_{2}}\left(X^{x}\left(t_{2}\right)\right) \mid X^{x}\left(t_{1}\right)\right)\right] \\
& =E \sum_{i_{1} \in A_{1}} \sum_{i_{2} \in A_{2}} \nu_{i_{1}}\left(X^{x}\left(t_{1}\right)\right) \nu_{i_{2}}\left(X^{x}\left(t_{2}\right)\right) .
\end{aligned}
$$

In fact, by induction, we obtain the finite-dimensional distributions

$$
\begin{aligned}
& P\left(\alpha^{\varepsilon, x, i}\left(t_{1}\right) \in A_{1}, \alpha^{\varepsilon, x, i}\left(t_{2}\right) \in A_{2}, \ldots, \alpha^{\varepsilon, x, i}\left(t_{n}\right) \in A_{n}\right) \\
& \quad \rightarrow E \sum_{i_{1} \in A_{1}} \sum_{i_{2} \in A_{2}} \cdots \sum_{i_{n} \in A_{n}} \nu_{i_{1}}\left(X^{x}\left(t_{1}\right)\right) \nu_{i_{2}}\left(X^{x}\left(t_{2}\right)\right) \cdots \nu_{i_{n}}\left(X^{x}\left(t_{n}\right)\right) \text { as } \varepsilon \rightarrow 0 .
\end{aligned}
$$

As another illustration, consider a control problem with the cost function given by

$$
J^{\varepsilon}(x, \alpha, u(\cdot))=E_{x, \alpha} \int_{0}^{T} C\left(X^{\varepsilon}(t), \alpha^{\varepsilon}(t), u(t)\right) d t,
$$

where $\left(X^{\varepsilon}(\cdot), \alpha^{\varepsilon}(\cdot)\right)$ is given by (5.66). Generally, the problem is difficult to solve due to the complexity of the problem setup. Using our asymptotic expansions, we can show that there is an associated cost function for the limit problem

$$
J(x, u(\cdot))=E_{x} \int_{0}^{T} \bar{C}(X(t), u(t)) d t
$$

where $X(t)$ is given in (5.67) and $\bar{C}(x, u)=\sum_{i=1}^{m} C(x, i) \nu_{i}(x)$ as defined in (5.68). We

can then find optimal control of the limit problem. Using this optimal control in the original system, we can obtain asymptotic optimal control under suitable conditions.

\section{REFERENCES}

[1] G. Barone-Adesi and R. Whaley, Efficient analytic approximation of American option values, $J$. Finance, 42 (1987), 301-320.

[2] N.N. Bogoliubov and Y.A. Mitropolsky, Asymptotic Methods in the Theory of Non-linear Oscillations, Gordon and Breach, New York, 1961. MR0141845 (25:5242)

[3] L.C. Evans, Partial Differential Equations, American Mathematical Society, 1998. MR.1625845 (99e:35001)

[4] J.P. Fouque, G. Papanicolaou, and R.K. Sircar, Derivatives in Financial Markets with Stochastic Volatility, Cambridge University Press, 2000. MR 1768877 (2002g:91082)

[5] A.M. Il'in, R.Z. Khasminskii, and G. Yin, Singularly perturbed switching diffusions: rapid switchings and fast diffusions, J. Optim. Theory Appl., 102 (1999), 555-591. MR1710722 (2000m:60071)

[6] A.M. Il'in, R.Z. Khasminskii, and G. Yin, Asymptotic expansions of solutions of integro-differential equations for transition densities of singularly perturbed switching diffusions: Rapid switchings, $J$. Math. Anal. Appl., 238 (1999), 516-539. MR.1715497(2001f:60083)

[7] R.Z. Khasminskii and G. Yin, On averaging principles: An asymptotic expansion approach, SIAM J. Math. Anal., 35 (2004), 1534-1560. MR2083789 (2005h:60245) 
[8] R.Z. Khashinskii, G. Yin, and Q. Zhang, Asymptotic Expansions of Singularly Perturbed Systems Involving Rapidly Fluctuating Markov Chains, SIAM Journal on Applied Mathematics, 56 (1996), 277-293. MR 1372901 (97d:34066)

[9] R. Z. Khasminskii and G. Yin, Asymptotic Series for Singularly Perturbed Kolmogorov-FokkerPlanck Equations, SIAM Journal on Applied Mathematics, 56 (1996), 1766-1793. MR1417479 (97j:60141)

[10] R.G. Phillips and P.V. Kokotovic, A singular perturbation approach to modeling and control of Markov chains, IEEE Trans. Automat. Control, 26 (1981), 1087-1094.

[11] S.P. Sethi and Q. Zhang, Hierarchical Decision Making in Stochastic Manufacturing Systems, Birkhäuser, Boston, MA, 1994. MR1301778 (95j:90006)

[12] H.A. Simon and A. Ando, Aggregation of variables in dynamic systems, Econometrica, 29 (1961), 111-138.

[13] G. Yin and X.Y. Zhou, Markowitz's mean-variance portfolio selection with regime switching: from discrete-time models to their continuous-time limits, IEEE Trans. Automat. Control, 49 (2004), 349-360. MR 2062248 (2005b:91139)

[14] G. Yin and Q. Zhang, Continuous-time Markov Chains and Applications: A Singular Perturbation Approach, Springer-Verlag, New York, NY, 1998. MR1488963 (2000a:60142)

[15] G. Yin, Q. Zhang, and G. Badowski, Asymptotic properties of a singularly perturbed Markov chain with inclusion of transient states, Ann. Appl. Probab., 10 (2000), 549-572. MR1768223 (2002c:60123)

[16] G. Yin and C. Zhu, Hybrid Switching Diffusions: Properties and Applications, Springer, New York, 2010. MR 2559912(2010i:60226) 